\title{
Appell Transformation and Canonical Transforms ${ }^{\star}$
}

\author{
Amalia TORRE
}

ENEA UTAPRAD-MAT Laboratorio di Modellistica Matematica, via E. Fermi 45, 00044 Frascati (Rome), Italy

E-mail: amalia.torre@enea.it

Received January 31, 2011, in final form July 11, 2011; Published online July 19, 2011 doi:10.3842/SIGMA.2011.072

\begin{abstract}
The interpretation of the optical Appell transformation, as previously elaborated in relation to the free-space paraxial propagation under both a rectangular and a circular cylindrical symmetry, is reviewed. Then, the caloric Appell transformation, well known in the theory of heat equation, is shown to be amenable for a similar interpretation involving the Laplace transform rather than the Fourier transform, when dealing with the $1 \mathrm{D}$ heat equation. Accordingly, when considering the radial heat equation, suitably defined Hankeltype transforms come to be involved in the inherent Appell transformation. The analysis is aimed at outlining the link between the Appell transformation and the canonical transforms.
\end{abstract}

Key words: heat equation; paraxial wave equation; Appell transformation

2010 Mathematics Subject Classification: 35K05; 35K10; 47D06

\section{Introduction}

The Appell transformation [1] is mainly associated with the heat equation (HE) $[1,2,3,4,5,6,7]$. Some recent analyses however have shown its relevance in other contexts as well $[8,9]$.

In [9] some issues related to the 1D HE have been revisited within the context of the free-space paraxial propagation, formally accounted for by the 2D paraxial wave equation (PWE). Thus, the Appell transformation has been interpreted in the light of the propagation of given source functions, which are in a definite relation with the source functions of the original wavefunctions (i.e. solutions of the PWE), relatively to both a rectangular and a circular cylindrical symmetry.

The analysis has been developed by following the Lie-algebra based approach to evolution equations, ruled by Hamiltonian operators underlying a harmonic oscillator-like symmetry algebra, as originally elaborated in a series of seminal papers by Kalnins, Miller and Boyer [10, 11].

In fact, the 1D HE and the 2D PWE as well as the $(1+1) \mathrm{D}$ Schrödinger equation (SE) can be considered as evolution equations, ruled by Hamiltonian-like operators (not necessarily Hermitian) which are quadratic in the inherent canonically conjugate variables.

The 1D HE has been the object of deep investigations [1, 2, 3, 4, 5, 6, 7, 8, 9], with the relevant Appell transformation being also embedded in the Lie symmetry group [12, 13, 14]. Extensive studies have been devoted to the PWE and the SE as well. In [15] and [16], for instance, the aforementioned Lie algebra based method has been systematically applied to the 2D PWE, and markedly interpreted in terms of solutions obtained by propagating definite "source functions", identified according to that method as eigenstates of specific operators in the inherent symmetry algebra $[10,11,12]$. Consequently, the transformations between wavefunctions can be seen to trace back to definite relations between the respective source functions, as explicitly shown in [17] through an analysis aimed at characterizing transformations between wavefunctions in terms of transformations between the relevant source functions.

\footnotetext{
*This paper is a contribution to the Special Issue "Symmetry, Separation, Super-integrability and Special Functions $\left(\mathrm{S}^{4}\right)$ ". The full collection is available at http://www.emis.de/journals/SIGMA/S4.html
} 
In particular, the optical Appell transformation represents the mapping between wavefunctions resulting from Fourier or Hankel pairs of source functions. This has been shown in [9] by proving the correspondence through the Appell transformation of wavefunctions generated by eigenstates of operators belonging to the PWE symmetry algebra, which are linked by a Fourier or Hankel similarity transformation, according to whether a rectangular or circular cylindrical geometry is concerned. As a mere consequence, the fractional Appell transformation has been introduced by involving the fractional Fourier or Hankel transform. Thus, a family of symmetry transformations for the 2D PWE, parameterized by a continuous parameter, has been identified.

Although a direct check of the result can easily be carried out, the formal steps followed through the analysis, as presented in [9] and reviewed below, have been aimed at favoring a "visualization" of the process, in view also of the possibility of suggesting a practical scheme for the realization of the optical "Appell transformer" as parallel to the optical Fourier transformer.

The purpose of the present paper is to show that a similar interpretation of the caloric Appell transformation can be elaborated, resorting to the bilateral Laplace transform rather than to the Fourier transform, as far as the 1D HE is concerned. We will frame the analysis within the Lie-algebra based formalism, as it - besides its intrinsic formal and conceptual elegance reveals effective in suggesting relations with other fields or theories. By retracing the same formal steps followed when dealing with the PWE, we will prove in fact that the caloric Appell transformation manifests the correspondence between temperature functions generated by eigenstates of operators belonging to the HE symmetry algebra, which are linked by a Laplacesimilarity transformation. Then, resorting to the definition of the fractional Laplace transform, a fractional caloric Appell transformation will as well be introduced.

Although, as earlier noted, the caloric Appell transformation has already been embedded in the Lie symmetry group $[12,13,14]$, to the author's knowledge, the Laplace-based relation of concern in the present context has still not been explicitly discussed in the literature. In our opinion, indeed, the parallelism between the HE and the PWE, which stems from the underlying algebra, is still not thoroughly exploited, whilst it may yield interesting results. For instance, the property, proven in [6], that the Appell transformation for an in general $n$-dimensional HE is essentially the only symmetry transformation (in the sense that every symmetry transformation can be obtained by composing Appell transformations with suitable scalings and shifts of all the variables) has been extended in [18] to the $2 \mathrm{D}$ PWE as well, by resorting to the symplectic ray-matrix formalism, usually adopted in paraxial optics. The stated property stems from the fact that the generators of the PWE symmetry algebra are Fourier or Hankel-similarity related.

Likewise, in regard to the radial $\mathrm{HE}$ we will prove that the relevant Appell transformation involves a similarity transformation by properly defined Hankel-type transforms.

As a result, one can recognize a direct link between the Appell transformation and the linear canonical transforms, since the (fractional) Fourier transform is an important representative of the real linear canonical transforms as the (fractional) bilateral Laplace transform is an equally important representative of the complex linear canonical transforms. Likewise, as we will see, the transforms, which relate to the Appell transformation for the radial HE, can be framed within the context of the radial canonical transforms.

In Section 2 we will firstly review the basics of the symmetry algebra-based method and later the results presented in [9], where the optical Appell transformation has been introduced and related to the Fourier or Hankel transform according to whether the PWE in rectangular or radial coordinate is concerned. In Section 3 the basics of the canonical transforms theory, specifically in relation with the bilateral Laplace and Hankel-type transforms, will be reviewed. Then, retracing the steps of the analysis presented in Section 2, we will prove in Section 4 that the caloric Appell transformation for the 1D HE connects temperature functions generated by eigenstates of operators in the inherent symmetry-algebra, which are linked through a Laplace-similarity transformation. A similar relation will be shown to hold also for the Appell transformation for 
the radial HE, accordingly resorting to suitably defined Hankel-type transforms, explicable as "radial-Laplace"-type transforms. Concluding comments will be given in Section 5.

\section{Optical Appell transformation: Fourier and Hankel transforms}

There is a well-known formal analogy between the $(2+1) \mathrm{D}$ SE, ruling the dynamics in two space-dimensions of a quantum particle under the action of some potential, and the 3D PWE, describing the paraxial propagation of a monochromatic scalar light-field through some medium, under the basic correspondences $t \rightarrow z, \hbar \rightarrow \lambda / 2 \pi$, and some others less direct, relating the momentum of the particle and the potential to the direction of propagation of the signal and the refractive index of the medium. Due to such an analogy, in fact, the Lie algebra-based method, as developed in a series of seminal papers by Kalnins, Miller and Boyer $[10,11,12]$ in connection with the solutions of the SE, has been applied as well to the free-space 2D PWE, both "linear" and "radial" $[15,16]$, on account in particular of the results in [10], pertaining the $(1+1) \mathrm{D} \mathrm{SE}$ respectively for a free particle and for a particle under a potential of the type $c / x^{2}$.

The free-space paraxial propagation is accounted for by the (free-space) 3D PWE, conveniently expressed in the normalized form:

$$
\left[2 i \frac{\partial}{\partial \zeta}+\frac{\partial^{2}}{\partial \xi^{2}}+\frac{\partial^{2}}{\partial \eta^{2}}\right] v(\xi, \eta, \zeta)=0
$$

the wavefunction $v(\xi, \eta, \zeta)$ being intended to belong to the complex space $\mathfrak{F}$ of locally $C^{\infty}$ functions of the real unitless variables $\xi, \eta, \zeta$. The latter represent the transverse and longitudinal (Cartesian) coordinates $x, y$, and $z$ scaled, respectively, to some characteristic transverse scale $w_{0}$, and to the relevant confocal parameter $b=k w_{0}^{2}, k$ being the wavenumber of the light.

Equation (2.1) admits separable-variable solutions in both Cartesian and polar coordinates, which are suitable to describe wavefunctions displaying respectively a rectangular and a circular cylindrical symmetry. In both cases one deals with a 2D equation, involving only one transverse coordinate, the longitudinal coordinate $\zeta$ playing the role of an evolution variable.

\section{$2.12 \mathrm{D}$ "linear" paraxial wave equation}

The separability of the solutions of (2.1) in rectangular coordinates manifests in the factorization of the $3 \mathrm{D}$ wavefunction $v(\xi, \eta, \zeta)$ in terms of two $2 \mathrm{D}$ wavefunctions $u(\xi, \zeta)$ and $w(\eta, \zeta)$ as

$$
v(\xi, \eta, \zeta)=u(\xi, \zeta) w(\eta, \zeta)
$$

each satisfying the 2D PWE in one transverse coordinate:

$$
\left[2 i \frac{\partial}{\partial \zeta}+\frac{\partial^{2}}{\partial \xi^{2}}\right] u(\xi, \zeta)=0
$$

It is formally similar to the $(1+1) \mathrm{D}$ SE for the free-particle Hamiltonian $\widehat{\mathrm{H}}=-(1 / 2) \partial^{2} / \partial \xi^{2}$.

The relevant evolution operator (i.e. the paraxial propagator) is

$$
\widehat{\mathcal{U}}_{\mathrm{PWE}}(\zeta):=e^{i \frac{\zeta}{2} \frac{\partial^{2}}{\partial \xi^{2}}}
$$

so that solutions to $(2.2)$, for given initial conditions $u(\xi, 0)=u_{0}(\xi)$, are obtained as [19]

$$
u(\xi, \zeta)=\left[\widehat{\mathcal{U}}_{\mathrm{PWE}}(\zeta) u_{0}\right](\xi)=\frac{1}{\sqrt{2 \pi i \zeta}} \int_{-\infty}^{\infty} e^{i \frac{\left(\xi-\xi^{\prime}\right)^{2}}{2 \zeta}} u_{0}\left(\xi^{\prime}\right) d \xi^{\prime}
$$

under the minimal assumption that $u_{0}(\xi)$ tends to zero sufficiently rapidly as $\xi \rightarrow \pm \infty$. 
The above integral is well-known in paraxial optics as the Huygens-Fresnel (or, Collins) diffraction integral (specifically, for free-propagation) [20, 21].

Equation (2.3) implicitly assumes the equivalence of the representations of the propagator $\widehat{\mathcal{U}}_{\mathrm{PWE}}(\zeta)$ as a Fresnel transform and as an exponential operator, involving the free-Hamiltonian. Evidently, the former is meaningful only if $u_{0}(\xi)$ is integrable and the pertinent integral converges, whereas the latter requires the implied series of derivatives $\frac{\partial^{2 n} u_{0}}{\partial \xi^{2 n}}$ to exist and converge to a finite value as well. We will not dwell here on the legitimacy of such an equivalence, but we will simply assume to deal with functions for which it holds [19].

\subsubsection{Solving the PWE: the Kalnins-Miller-Boyer method}

The symmetry-algebra based approach, as developed in [10, 11, 12], identifies a systematic method to solve (2.2), based on the spectral decomposition of operators belonging to the inherent symmetry algebra $\mathcal{G}$. This is the semidirect sum of the algebra $s l(2, \mathbb{R}) \simeq s p(2, \mathbb{R}) \simeq s u(1,1)$, spanned by the operators $\left\{\widehat{\mathrm{K}}_{+}, \widehat{\mathrm{K}}_{3}, \widehat{\mathrm{K}}_{-}\right\}$, with

$$
\begin{aligned}
& \widehat{\mathrm{K}}_{+}:=\frac{1}{2} \xi^{2}, \quad \widehat{\mathrm{K}}_{3}:=-\frac{i}{2}\left(\xi \frac{\partial}{\partial \xi}+\frac{1}{2}\right), \quad \widehat{\mathrm{K}}_{-}:=-\frac{1}{2} \frac{\partial^{2}}{\partial \xi^{2}} \\
& {\left[\widehat{\mathrm{K}}_{+}, \widehat{\mathrm{K}}_{-}\right]=2 i \widehat{\mathrm{K}}_{3}, \quad\left[\widehat{\mathrm{K}}_{ \pm}, \widehat{\mathrm{K}}_{3}\right]= \pm i \widehat{\mathrm{K}}_{ \pm},}
\end{aligned}
$$

and the Weyl algebra $\mathcal{W}$, spanned by $\{\widehat{\mathrm{X}}, \widehat{\mathrm{P}}, \widehat{\mathrm{I}}\}$, such that

$$
\widehat{\mathrm{X}}:=\xi, \quad \widehat{\mathrm{P}}:=-i \frac{\partial}{\partial \xi}, \quad \widehat{\mathrm{I}}=1, \quad[\widehat{\mathrm{X}}, \widehat{\mathrm{P}}]=i \widehat{\mathrm{I}}
$$

The algebra $\mathcal{G}=\mathcal{W} \oplus \operatorname{sl}(2, \mathbb{R})$ is sometimes referred to in the literature as $w \operatorname{sl}(2, \mathbb{R}) ; \mathcal{W}$ is an ideal in $w s l(2, \mathbb{R})$, as conveyed by the mixed commutators,

$$
\begin{aligned}
& {\left[\widehat{\mathrm{K}}_{+}, \widehat{\mathrm{X}}\right]=0, \quad\left[\widehat{\mathrm{K}}_{+}, \widehat{\mathrm{P}}\right]=i \widehat{\mathrm{X}}, \quad\left[\widehat{\mathrm{K}}_{-}, \widehat{\mathrm{X}}\right]=-i \widehat{\mathrm{P}}, \quad\left[\widehat{\mathrm{K}}_{-}, \widehat{\mathrm{P}}\right]=0} \\
& {\left[\widehat{\mathrm{K}}_{3}, \widehat{\mathrm{X}}\right]=-\frac{i}{2} \widehat{\mathrm{X}}, \quad\left[\widehat{\mathrm{K}}_{3}, \widehat{\mathrm{P}}\right]=\frac{i}{2} \widehat{\mathrm{P}}, \quad\left[\widehat{\mathrm{K}}_{ \pm, 3}, \widehat{\mathrm{I}}\right]=0}
\end{aligned}
$$

The operators (2.4) and (2.5) are self-adjoint when acting on the Hilbert space $\mathfrak{L}^{2}(\mathbb{R})$ of complex-valued Lebesgue square-integrable functions on the real line $\mathbb{R}$ with the scalar product $\langle f, g\rangle \equiv \int_{-\infty}^{+\infty} f(\xi) g^{*}(\xi) d \xi$, the star denoting complex conjugation.

As formulated in [10], the spectral decomposition $\left\{f_{\lambda}\right\}_{\lambda}$ of any $\widehat{\mathrm{K}} \in \mathcal{G}$,

$$
\widehat{\mathrm{K}} f_{\lambda}(\xi)=\lambda f_{\lambda}(\xi),
$$

can be used to construct solutions of the PWE having the $f_{\lambda} \mathrm{s}$ as initial conditions.

This can be done by evaluating the Huygens-Fresnel integral (2.3) for each $f_{\lambda}$, provided it be in the domain of $\widehat{\mathrm{K}}_{-}$, thus obtaining the propagated functions $v_{\lambda}(\xi, \zeta)=\widehat{\mathcal{U}}_{\mathrm{PWE}}(\zeta) f_{\lambda}(\xi)$.

Alternatively, one can search for the solutions of the equations

$$
\widehat{\mathrm{K}}(\zeta) v_{\lambda}(\xi, \zeta)=\lambda v_{\lambda}(\xi, \zeta)
$$

which directly follow from $(2.6)$ on account of $f_{\lambda}(\xi)=\widehat{\mathcal{U}}_{\mathrm{PWE}}(\zeta)^{-1} v_{\lambda}(\xi, \zeta)$, and hence address the initial conditions $v_{\lambda}(\xi, 0)=f_{\lambda}(\xi)$. The operator $\widehat{\mathrm{K}}(\zeta)$, given by

$$
\widehat{\mathrm{K}}(\zeta):=e^{-i \zeta \widehat{\mathrm{K}}_{-}} \widehat{\mathrm{K}} e^{i \zeta \widehat{\mathrm{K}}_{-}},
$$

can be understood as a Heisenberg-like operator corresponding to $\widehat{K}$. In fact, the above mapping, which relates "fixed-location" operators $\widehat{\mathrm{K}} \in \mathcal{G}$ to "evolving-location" operators $\widehat{\mathrm{K}}(\zeta) \in \mathcal{G}$, 
is reminiscent of the transformation relating the Heisenberg and Schrödinger pictures of quantum mechanics (involving $\mathfrak{L}^{2}(\mathbb{R})$-wavefunctions) ${ }^{1}$. More precisely, the operator $\widehat{\mathrm{K}}(\zeta)$ should be regarded as the "back-evolving location" form of $\widehat{\mathrm{K}}$.

Since

$$
e^{\widehat{\mathrm{A}}} \widehat{\mathrm{B}} e^{-\widehat{\mathrm{A}}}=\sum_{j=0}^{\infty}(\operatorname{ad} \widehat{\mathrm{A}})^{j} \widehat{\mathrm{B}} / j !, \quad \text { with } \quad(\operatorname{ad} \widehat{\mathrm{A}})^{j} \widehat{\mathrm{B}}=[\widehat{\mathrm{A}},[\ldots,[\widehat{\mathrm{A}},[\widehat{\mathrm{A}}, \widehat{\mathrm{B}}]]]]
$$

being a $j$-fold commutator, the $\widehat{\mathrm{K}}(\zeta)$ s are finite linear combinations of the fixed-location operators (2.4) and (2.5) with coefficients depending on the propagation variable $\zeta$, which so enters $(2.7)$ as a parameter.

Evidently, the above sketched procedure, based on the eigenvalue equations (2.6) or (2.7), is equivalent to a separation of variables; in fact, in [10] a well-defined connection between separation of variables and Lie symmetries for (2.2) has been established (see also [12]).

In [15], the procedure has markedly been interpreted in terms of wavefunctions obtained by propagating definite "source functions", identified as eigenstates of specific operators in $\mathcal{G}$. Accordingly, the transformations between wavefunctions trace back to definite relations between the respective source functions [17]. Further cases besides those considered in [10] have been analyzed in [15]. Thus, a certain class of solutions of (2.2) has been obtained by propagating the eigenfunctions of the operator $\widehat{\mathrm{K}}_{\xi_{0}}=\widehat{\mathrm{K}}_{3}-\left(2 / \xi_{0}\right) \widehat{\mathrm{K}}_{+}$, $\xi_{0}$ being an arbitrary parameter. Such solutions depend on three independent parameters and basically comprise a complex quadratic exponential modulated by the Weber-Hermite function $D_{-2 i \lambda-1 / 2}$ of suitable argument.

The same result was previously deduced in [23] on the basis of an ansatz giving the general solution of the 2D PWE in a suitable separable-variable form. A similar ansatz have led the same authors to identify general solutions of (2.1) pertaining to a circular cylindrical symmetry [24]; the elliptical cylindrical symmetry has been considered in [25].

On the other hand, as shown in [17], a definite relation between the wavefunctions arising from the eigenfunctions of the operators $\widehat{\mathrm{K}}_{\xi_{0}}$ and $\widehat{\mathrm{K}}_{3}$ can be established, the former following from the latter under the symmetry transformation produced by the operator $\exp \left[\left(i / \xi_{0}\right) \widehat{\mathrm{K}}_{+}(\zeta)\right]$.

\subsubsection{Fourier transform and optical Appell transformation}

As proven in [9], the optical Appell transformation connects wavefunctions, whose source functions are Fourier related. In order to review this result, we consider for every operator $\widehat{\mathrm{K}} \in \mathcal{G}$ the dual operator $\widehat{\widetilde{\mathrm{K}}}$, linked to the former through a similarity transformation by the direct or inverse Fourier transform operator $\widehat{\mathcal{F}}$, i.e. $\widehat{\widetilde{K}}=\widehat{\mathcal{F}} \widehat{\mathrm{K}} \widehat{\mathcal{F}}^{-1}$ or $\widehat{\widetilde{K}}=\widehat{\mathcal{F}}^{-1} \widehat{\mathrm{K}} \widehat{\mathcal{F}}$, both belonging to $\mathcal{G}$.

The Fourier transform, which is well known to operate as

$$
\widetilde{f}(x)=[\widehat{\mathcal{F}} f](x):=\frac{1}{\sqrt{2 \pi}} \int_{-\infty}^{+\infty} e^{-i x x^{\prime}} f\left(x^{\prime}\right) d x^{\prime}
$$

admits the exponential operator representation in terms of the $s u(1,1)$ generators $\widehat{\mathrm{K}}_{+}$and $\widehat{\mathrm{K}}_{-}$:

$$
\widehat{\mathcal{F}}=e^{i \pi / 4} e^{-i(\pi / 2)\left[\widehat{\mathrm{K}}_{-}+\widehat{\mathrm{K}}_{+}\right]}
$$

the factor $e^{i \pi / 4}$ allowing the optical transform to be matched to the mathematical transform.

${ }^{1}$ The Heisenberg picture of quantum mechanics amounts to the observable [22]

$$
\widehat{\mathrm{O}}(t)=\widehat{\mathcal{U}}^{\dagger}(t) \widehat{\mathrm{O}} \widehat{\mathcal{U}}(t),
$$

where $\widehat{\mathcal{U}}(t)$ is the unitary evolution operator, which obeys the relations $\widehat{\mathcal{U}}^{\dagger}(t)=\widehat{\mathcal{U}}^{-1}(t)$ and $\widehat{\mathcal{U}}^{-1}(t)=\widehat{\mathcal{U}}(-t)$. 
By optical transform we mean the linear canonical transform resulting from the map of the symplectic group $S p(2, \mathbb{R})(\simeq S L(2, \mathbb{R}))$ into the metaplectic group $M p(2, \mathbb{R})$, which turns optical ray-tracing matrices $\mathbf{M}=\left(\begin{array}{ll}A & B \\ C & D\end{array}\right)$ in the former into the Collins integral in the latter as [20, 21]

$$
v\left(\xi, \zeta_{o}\right)=\widehat{\mathcal{U}}_{\left(\begin{array}{cc}
A & B \\
C & D
\end{array}\right)} v\left(\xi, \zeta_{i}\right)=\frac{1}{\sqrt{2 \pi i B}} \int_{-\infty}^{+\infty} \exp \left\{\frac{i}{2 B}\left(A \xi^{\prime 2}-2 \xi \xi^{\prime}+D \xi^{2}\right)\right\} v\left(\xi^{\prime}, \zeta_{i}\right) d \xi^{\prime} .
$$

The optical system described by the ray-matrix $\mathbf{M}$ is therefore seen as an operator transporting the wavefunction on the input plane located at $\zeta_{i}$ to the wavefunction on the output plane at $\zeta_{o}$. The metaplectic group $M p(2, \mathbb{R})$ provides a double cover of the symplectic group $S p(2, \mathbb{R})$, to which the Ms belong. In fact, although not explicitly displayed by convention, a double sign \pm is implied in $(2.10)^{2}$, thus allowing the metaplectic images of the Ms to close into a group.

It may be useful to clarify that throughout the paper $\sqrt{i}$ will be intended to identify the principal square root of the imaginary unit: $\sqrt{i}=e^{i \pi / 4}$, as it will be in general for the square root of any (complex) number: $\sqrt{a}=\sqrt{|a|} e^{i \arg (a) / 2}$, with $\arg (a) \in(-\pi, \pi]$.

The diffraction integral (2.3) follows from (2.10) in correspondence to the free-section matrix

$$
\mathbf{T}(\zeta)=\left(\begin{array}{ll}
1 & \zeta \\
0 & 1
\end{array}\right)
$$

i.e. $\widehat{\mathcal{U}}_{\mathrm{PWE}}(\zeta)=\widehat{\mathcal{U}}_{\left(\begin{array}{cc}1 & \zeta \\ 0 & 1\end{array}\right)}$.

In virtue of the disentanglement relation for the $s u(1,1)$ algebra generators

$$
e^{i \beta\left[\widehat{\mathrm{K}}_{-}+\widehat{\mathrm{K}}_{+}\right]}=e^{i \tan (\beta / 2) \widehat{\mathrm{K}}_{-}} e^{i \sin (\beta) \widehat{\mathrm{K}}_{+}} e^{i \tan (\beta / 2) \widehat{\mathrm{K}}_{-}}=e^{i \tan (\beta / 2) \widehat{\mathrm{K}}_{+}} e^{i \sin (\beta) \widehat{\mathrm{K}}_{-}} e^{i \tan (\beta / 2) \widehat{\mathrm{K}}_{+}},
$$

holding for $|\beta|<\pi$, the exponential operator in (2.9) is factorizable in the two equivalent forms

$$
e^{-i(\pi / 2)\left[\widehat{\mathrm{K}}_{-}+\widehat{\mathrm{K}}_{+}\right]}=e^{-i \widehat{\mathrm{K}}_{-}} e^{-i \widehat{\mathrm{K}}_{+}} e^{-i \widehat{\mathrm{K}}_{-}}=e^{-i \widehat{\mathrm{K}}_{+}} e^{-i \widehat{\mathrm{K}}_{-}} e^{-i \widehat{\mathrm{K}}_{+}} .
$$

They reproduce the two possible implementations of the Fourier transform by optical elements, i.e. the $2 f$-system and the Fourier tube, respectively consisting of a single lens (of focal length $f$ ) placed midway between two reference planes separated by $2 f$ and of two identical lenses (of focal length $f$ ) separated by $f$. Both setups are described by the ray-matrix $\mathbf{F}(f)=\left(\begin{array}{cc}0 & f \\ -1 / f & 0\end{array}\right)$; in particular, $f=1$ in (2.9) and so in (2.13). Unless otherwise specified, we will address to as Fourier transformer the optical system described by the ray-matrix $\mathbf{F}(1)=\mathbf{F}=\left(\begin{array}{rr}0 & 1 \\ -1 & 0\end{array}\right)$.

Since every $\widehat{\mathrm{K}} \in \mathcal{G}$ is a linear combination of the basis operators (2.4) and (2.5), by (2.13) also $\widehat{\mathcal{F}} \widehat{K} \widehat{\mathcal{F}}^{-1}$ and $\widehat{\mathcal{F}}^{-1} \widehat{\mathrm{K}} \widehat{\mathcal{F}}$ are linear combinations of the same operators, and hence belong to $\mathcal{G}$.

Then, as parallel to the eigenvalue problem for the operator $\widehat{K}$, signified by (2.6) and (2.7), we consider the eigenvalue problem for the dual operator $\widehat{\widetilde{K}}$, expressed by the equation

$$
\widehat{\widetilde{\mathrm{K}}} g_{\lambda}(\xi)=\lambda g_{\lambda}(\xi)
$$

for the fixed-location operator, and by

$$
\widehat{\widetilde{\mathrm{K}}}(\zeta) w_{\lambda}(\xi, \zeta)=\lambda w_{\lambda}(\xi, \zeta), \quad \widehat{\widetilde{\mathrm{K}}}(\zeta)=e^{-i \zeta \widehat{\mathrm{K}}_{-}} \widehat{\widehat{\mathrm{K}}} e^{i \zeta \widehat{\mathrm{K}}_{-}},
$$

for the relevant evolving-location operator.

Let us suppose that $\widehat{\widetilde{K}}=\widehat{\mathcal{F}} \widehat{\mathrm{K}} \widehat{\mathcal{F}}^{-1}$. Accordingly, we see that

$$
g_{\lambda}=\widehat{\mathcal{F}} f_{\lambda},
$$

\footnotetext{
${ }^{2}$ A double sign \pm is accordingly implied also in the matching factor entering (2.9).
} 
and hence the $w_{\lambda}$ s are solutions of (2.2) obtained by propagating the Fourier transformed eigenfunctions of $\widehat{\mathrm{K}}$. Precisely, the $w_{\lambda} \mathrm{s}$ are obtained from the $v_{\lambda} \mathrm{s}$ through the "local" transformation

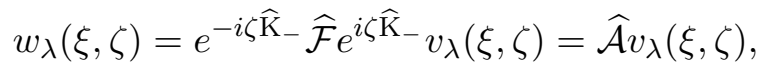

by the symmetry operator

$$
\widehat{\mathcal{A}}:=e^{-i \zeta \widehat{\mathrm{K}}_{-}} \widehat{\mathcal{F}} e^{i \zeta \widehat{\mathrm{K}}_{-}},
$$

addressed to in [9] as Appell transform operator. In the light of (2.8), it can be understood as a (back) evolving-location Fourier operator: $\widehat{\mathcal{A}}=\widehat{\mathcal{F}}(\zeta)$. It depends on the evolution variable $\zeta$; however, such a dependence will not be explicitly displayed.

As noted in [9], the Appell operator (2.14) individualizes the optical ABCD system, which arises from the composition of a Fourier transformer embedded between two free-space sections of length $-\zeta$ and $\zeta$. It is therefore described by the symplectic real ray-matrix

$$
\mathbf{M}_{\widehat{\mathcal{A}}}=\left(\begin{array}{ll}
1 & \zeta \\
0 & 1
\end{array}\right)\left(\begin{array}{cc}
0 & 1 \\
-1 & 0
\end{array}\right)\left(\begin{array}{cc}
1 & -\zeta \\
0 & 1
\end{array}\right)=\left(\begin{array}{cc}
-\zeta & 1+\zeta^{2} \\
-1 & \zeta
\end{array}\right)
$$

according to the $2 \times 2$ matrix representation of each relevant optical component (i.e. operator) ${ }^{3}$.

As a more explicit form of the Collins integral (2.10), the propagation of a signal through an $\mathrm{ABCD}$ system manifests in the transformation of the relevant wavefunction as [17, 27]:

$$
u\left(\xi, \zeta_{o}\right)=\widehat{\mathcal{U}}_{\left(\begin{array}{ll}
A & B \\
C & D
\end{array}\right)} u\left(\xi, \zeta_{i}\right)=\frac{1}{\sqrt{A}} e^{i \frac{C}{2 A} \xi^{2}} u\left(\frac{\xi}{A}, \zeta_{i}+\frac{B}{A}\right) .
$$

It signifies that the whole action of the system is decomposed $\grave{a}$ la Wei-Norman [28] into a sequence of a free-propagation by $B / A$, a lensing by focal power $-C$, and a scaling by $1 / A$. In fact, $u\left(\xi, \zeta_{i}+B / A\right)=\left[\widehat{\mathcal{U}}_{\mathrm{PWE}}\left(\zeta_{i}+B / A\right) u_{0}\right](\xi)$ represents the propagated form of the (effective or fictitious) source function $u_{0}(\xi)$ with which the wavefunction $u\left(\xi, \zeta_{i}\right)$ can be associated. Note that, since we are working with unitless variables, the off-diagonal entries $B$ and $C$ (usually having the dimensions of length and $1 /$ length) are intended to be normalized to $b$ and $1 / b$.

Relation (2.16) holds for $A \neq 0$. If $A=0$, according to (2.10) the wavefunction transforms as

$$
\widehat{\mathcal{U}}_{\left(\begin{array}{ll}
0 & B \\
C & D
\end{array}\right)} u\left(\xi, \zeta_{i}\right)=\frac{1}{\sqrt{i B}} e^{i \frac{D}{2 B} \xi^{2}} \widetilde{u}\left(\frac{\xi}{B}, \zeta_{i}\right),
$$

describing the effect of an optical Fourier transformer with focal length $f=B$ combined with a modulation by the phase factor $\exp \left(i \frac{D}{2 B} \xi^{2}\right)$.

By (2.16) we can express the associated functions $w_{\lambda}$ in terms of the $v_{\lambda}$ s according to Appell's prescription:

$$
w_{\lambda}(\xi, \zeta)=\frac{1}{\sqrt{i \zeta}} e^{i \frac{\xi^{2}}{2 \zeta}} v_{\lambda}\left(-\frac{\xi}{\zeta},-\frac{1}{\zeta}\right) .
$$

In the case of $\mathbf{M}_{\widehat{\mathcal{A}}}, A=0$ means $\zeta=0$, which amounts to $D=0$ and $B=1$. Hence, following (2.17) one recovers the primary relation between the source functions: $w_{\lambda}(\xi, 0)=$ $\widetilde{v}_{\lambda}(\xi, 0)$.

As noted in [9], relation (2.18) can also be deduced by acting on the initial function $v_{0}(\xi)$ by the operator $e^{-i \zeta \widehat{\mathrm{K}}_{-}} \widehat{\mathcal{F}}$, and hence (apart from $\left.\sqrt{i}\right)$ through $(2.16)$ with $\zeta_{i}=0$ and $\left(\begin{array}{ll}A & B \\ C & D\end{array}\right)=\left(\begin{array}{cc}-\zeta & 1 \\ -1 & 0\end{array}\right)$.

\footnotetext{
${ }^{3}$ For the practical realization of such a system by basic optical elements, as noted above, one may resort to free-sections and lenses for both the free-propagation by $\zeta$ and the Fourier transformer. According to the analysis in [26], the propagation by the "negative" distance $-\zeta$ may be realized by free-sections and lenses as well, specifically by a sequence of three suitably designed lenses separated by free-sections of proper lengths.
} 
If in turn one has $\widehat{\widetilde{K}}=\widehat{\mathcal{F}}^{-1} \widehat{\mathrm{K}} \widehat{\mathcal{F}}$, then

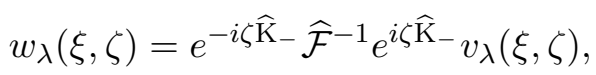

involving just the inverse of the Appell operator (2.14), i.e.

$$
\widehat{\mathcal{A}}^{-1}=e^{-i \zeta \widehat{\mathrm{K}}_{-} \widehat{\mathcal{F}}^{-1}} e^{i \zeta \widehat{\mathrm{K}}_{-}}=\widehat{\mathcal{F}}^{-1}(\zeta) .
$$

It individualizes the $\mathrm{ABCD}$ system

$$
\mathbf{M}_{\widehat{\mathcal{A}}^{-1}}=\left(\begin{array}{ll}
1 & \zeta \\
0 & 1
\end{array}\right)\left(\begin{array}{cc}
0 & -1 \\
1 & 0
\end{array}\right)\left(\begin{array}{cc}
1 & -\zeta \\
0 & 1
\end{array}\right)=\left(\begin{array}{cc}
\zeta & -1-\zeta^{2} \\
1 & -\zeta
\end{array}\right)=\mathbf{M}_{\widehat{\mathcal{A}}}^{-1},
$$

thus yielding the explicit transformation of the wavefunctions $v_{\lambda} \mathrm{s}$ into the associated $w_{\lambda} \mathrm{S}$ as

$$
w_{\lambda}(\xi, \zeta)=\frac{1}{\sqrt{i \zeta}} e^{i \frac{\xi^{2}}{2 \zeta}} v_{\lambda}\left(\frac{\xi}{\zeta},-\frac{1}{\zeta}\right) .
$$

As a conclusion, we may say that in general the optical Appell transformation

$$
w(\xi, \zeta)=\frac{1}{\sqrt{i \zeta}} e^{i \frac{\xi^{2}}{2 \zeta}} v\left( \pm \frac{\xi}{\zeta},-\frac{1}{\zeta}\right),
$$

maps solutions of the linear 2D PWE into solutions [9]. It is a symmetry transformation for that equation, which traces back to a (direct or inverse) Fourier relation between the source functions of the solutions it connects. It manifests the action of the evolving-location Fourier operator $\widehat{\mathcal{F}}(\zeta)$ or its inverse.

Note that the transformation (2.21) amounts to a mathematical Fourier relation between the source functions of the involved solutions. Indeed, an optical Fourier relation between the source functions would demand for the trivial change of the phase factor as $e^{-i \pi / 4} \rightarrow e^{-i \pi / 2}$.

As a basic example, we may consider the operators $\widehat{\mathrm{P}}$ and $\widehat{\mathrm{X}}=\widehat{\mathcal{F}} \widehat{\mathrm{P}} \widehat{\mathcal{F}}^{-1}$. The eigenfunctions of $\widehat{\mathrm{P}}$, i.e. the plane waves $f_{\lambda}(\xi)=\exp (i \lambda \xi) / \sqrt{2 \pi}, \lambda \in \mathbb{R}$, yield the wavefunctions

$$
v_{\lambda}(\xi, \zeta)=\frac{1}{\sqrt{2 \pi}} e^{i \lambda \xi} e^{-i \lambda^{2} \zeta / 2},
$$

which display the familiar frequency-chirping factor $\exp \left(-i \lambda^{2} \zeta / 2\right)$.

Applying the Appell transformation (2.18) to (2.22), one just obtains the wavefunctions

$$
w_{\lambda}(\xi, \zeta)=\frac{1}{\sqrt{2 \pi i \zeta}} e^{i \frac{(\xi-\lambda)^{2}}{2 \zeta}}
$$

one would obtain by propagating the eigenfunctions $g_{\lambda}(\xi)=\delta(\xi-\lambda), \lambda \in \mathbb{R}$, of the operator $\widehat{\mathrm{X}}$.

Vice versa, the wavefunctions (2.23) turn into the propagating plane waves (2.22) by $(2.20)$.

Another example of Appell pair of wavefunctions is provided by the two kinds of Airy beams

$$
\begin{aligned}
& \psi_{\mathrm{KM}}(\xi, \zeta, \lambda)=\frac{1}{\sqrt{i \zeta}} e^{i\left(\frac{1}{12 \zeta^{3}}+\frac{\xi^{2}}{2 \zeta}-\frac{\xi}{2 \zeta^{2}}+\frac{\lambda}{2 \zeta}\right)} \operatorname{Ai}\left(\frac{\xi}{\zeta}-\frac{1}{4 \zeta^{2}}-\lambda\right), \\
& \psi_{\mathrm{BB}}(\xi, \zeta, \lambda)=e^{-i\left(\frac{\zeta^{3}}{12}-\frac{\zeta \xi}{2}+\frac{\lambda}{2} \zeta\right)} \mathrm{Ai}\left(\xi-\frac{\zeta^{2}}{4}-\lambda\right),
\end{aligned}
$$

Ai denoting the Airy function of the first kind [29]. As seen in [30], they are respectively obtained by propagating the eigenfunctions of the operators $\widehat{\mathcal{P}}=2 \widehat{\mathrm{K}}_{+}+\widehat{\mathrm{P}}$ and $\widehat{\mathcal{X}}=2 \widehat{\mathrm{K}}_{-}+\widehat{\mathrm{X}}=\widehat{\mathcal{F}} \widehat{\mathcal{P}} \widehat{\mathcal{F}}^{-1}$. Accordingly, the former originate from the cubic phase $\psi_{\mathrm{KM}}(\xi, 0, \lambda)=\exp \left(i \lambda \xi-i \xi^{3} / 3\right) / \sqrt{2 \pi}[10]$, as the latter from an Airy pattern: $\psi_{\mathrm{BB}}(\xi, 0, \lambda)=\operatorname{Ai}(\xi-\lambda)$ [31]; in both cases, $\lambda$ signifies the eigenvalue of the involved operator $\widehat{\mathcal{P}}$ or $\widehat{\mathcal{X}}$.

It is easily verified that by $(2.18) \psi_{\mathrm{KM}} \rightarrow \psi_{\mathrm{BB}}$, and vice versa by $(2.20) \psi_{\mathrm{BB}} \rightarrow \psi_{\mathrm{KM}}$.

In the literature, the $\psi_{\mathrm{BB}} \mathrm{s}$ are addressed to as Airy beams. Along with other similar Airy-related solutions of the PWE, they have attracted a great deal of interest (both analytical/numerical and experimental), due to their non-spreading nature and their tendency to freely accelerate; some pertinent titles are in [31, 32, 33, 34, 35, 36, 37, 38, 39, 40, 41]. 


\subsubsection{Fractional Appell transformation}

Conforming to the relevant generator $\widehat{\mathrm{K}}_{-}+\widehat{\mathrm{K}}_{+}$, the Fourier transform $\widehat{\mathcal{F}}$ can be understood as the evolution operator describing the dynamics of a quantum attractive oscillator (optically conveyed by a quadratic-index focusing medium) observed at "time" $\tau=\pi / 2$, the motion in the optical phase-plane ${ }^{4}$ resulting in a clockwise rotation by $\pi / 2$. Thereby, according to (2.14), in the optical phase-plane the Appell transform amounts to a $\pi / 2$-clockwise rotation preceded and followed respectively by a negative and positive $\xi$-shear.

As is well known, the Fourier transform $\widehat{\mathcal{F}}$ is a specific determination of the more general fractional Fourier transform $\widehat{\mathcal{F}}^{\alpha}$, signified by the integral $[42,43,44,45]$

$$
\left[\widehat{\mathcal{F}}^{\alpha} \varphi\right](x):=\sqrt{\frac{1-i \cot \phi}{2 \pi}} \int_{-\infty}^{+\infty} d x^{\prime} e^{\frac{i}{2 \sin \phi}\left(x^{\prime 2} \cos \phi+x^{2} \cos \phi-2 x x^{\prime}\right)} \varphi\left(x^{\prime}\right)
$$

the order $\alpha$ specifying the angle $\phi=\alpha \pi / 2$, or equivalently by the operator

$$
\widehat{\mathcal{F}}^{\alpha}=e^{i \alpha \pi / 4} e^{-i \alpha(\pi / 2)\left[\widehat{\mathrm{K}}_{-}+\widehat{\mathrm{K}}_{+}\right]}=(\widehat{\mathcal{F}})^{\alpha} .
$$

Again, the factor $e^{i \alpha \pi / 4}$ relates the mathematical and optical transforms, the latter being yielded by the Collins integral (2.10) in correspondence to the pertinent ray-matrix $\mathbf{F}^{\alpha}=\left(\begin{array}{c}\cos \phi \\ -\sin \phi \\ \sin \phi\end{array}\right)$ cos

Both expressions reveal the periodicity

$$
\widehat{\mathcal{F}}^{\alpha+4 j}=\widehat{\mathcal{F}}^{\alpha}, \quad j=0, \pm 1, \pm 2, \ldots,
$$

so that the range of $\alpha$ can be limited to the interval $(-2,2]$. Evidently, the ordinary transform $(2.9)$ is recovered with $\alpha=1$, i.e. $\phi=\pi / 2: \widehat{\mathcal{F}}^{1}=\widehat{\mathcal{F}}$. Also, $\widehat{\mathcal{F}}^{0}=\widehat{\mathrm{I}}$.

Paralleling $\widehat{\mathcal{F}}, \widehat{\mathcal{F}}^{\alpha}$ is interpreted as the evolution operator associated with the harmonic oscillator dynamics monitored continuously at "time" $\tau=\alpha \pi / 2=\phi$. Accordingly, the corresponding motion in the optical phase-plane amounts to a clockwise rotation by $\phi$.

As conveyed by (2.12), $\widehat{\mathcal{F}}^{\alpha}$ admits two operator factorizations, which reflect its optical realizations as "fractional" versions of the implementations of the ordinary transform. One has, in fact, $\widehat{\mathcal{F}}^{\alpha}=e^{i \phi / 2} e^{-i \tan (\phi / 2) \widehat{\mathrm{K}}_{-}} e^{-i \sin (\phi) \widehat{\mathrm{K}}_{+}} e^{-i \tan (\phi / 2) \widehat{\mathrm{K}}_{-}}$for the single lens realization (Lohmann type I setup [44]) and $\widehat{\mathcal{F}}^{\alpha}=e^{i \phi / 2} e^{-i \tan (\phi / 2) \widehat{\mathrm{K}}_{+}} e^{-i \sin (\phi) \widehat{\mathrm{K}}_{-}} e^{-i \tan (\phi / 2) \widehat{\mathrm{K}}_{+}}$for the two-lens realization (Lohmann type II setup [44]). The former comprises a thin lens of focal length $1 / \sin (\phi)$ placed midway between two reference planes spaced by $2 \tan (\phi / 2)$, whereas the latter realizes the dual configuration, composed by two thin lenses of focal length $\cot (\phi / 2)$ separated by $\sin (\phi)$.

It has been suggested in [9] to consider the fractional Appell transform operator $\widehat{\mathcal{A}}^{\alpha}$, which then, as fractional version of the operator $\widehat{\mathcal{A}}$, has been written in the form

$$
\widehat{\mathcal{A}}^{\alpha}:=e^{-i \zeta \widehat{\mathrm{K}}_{-}} \widehat{\mathcal{F}}^{\alpha} e^{i \zeta \widehat{\mathrm{K}}_{-}}=\widehat{\mathcal{F}}^{\alpha}(\zeta)
$$

whose dependence on $\zeta$ will not explicitly displayed. The ordinary operator (2.14) corresponds to $\alpha=1$, whilst the inverse is $\left(\widehat{\mathcal{A}}^{\alpha}\right)^{-1}=\widehat{\mathcal{A}}^{-\alpha}$, since $\left(\widehat{\mathcal{F}}^{\alpha}\right)^{-1}=\widehat{\mathcal{F}}^{-\alpha}$.

\footnotetext{
${ }^{4}$ Let us recall that the phase-plane (for systems with one degree of freedom) is understood as the Cartesian plane formed by the relevant canonically conjugate variables $(q, p)$, obeying the Poisson-bracket relation $\{q, p\}=1$.

The light-ray coordinates $(q, p)$ of geometrical optics are canonically conjugate variables. They span the optical phase-plane, where light rays are represented by points, and accordingly the ray propagation through optical systems corresponds to the "motion" of the relevant representative point. For instance, free-sections and thin lenses produce under paraxial propagation $q$ and $p$-shears in the phase-plane, i.e. translations of the ray representative point respectively in the $q$ and $p$ direction.

Note that, in accord with the normalization of the space variables here adopted, the ray-variables $(q, p)$ become $\left(\begin{array}{l}\xi \\ v\end{array}\right) \equiv\left(\begin{array}{l}q / w_{0} \\ k w_{0} p\end{array}\right)$, the ray optical momentum $p$, i.e. the angle relative to the $z$-axis, being scaled to the natural far-field divergence $\vartheta_{\infty}=1 / k w_{0}$, associated with a beam having $w_{0}$ as a characteristic width.
} 
The operator (2.25) individualizes the ABCD system, described by the matrix

$$
\mathbf{M}_{\widehat{\mathcal{A}}^{\alpha}}=\left(\begin{array}{ll}
1 & \zeta \\
0 & 1
\end{array}\right)\left(\begin{array}{cc}
\cos \phi & \sin \phi \\
-\sin \phi & \cos \phi
\end{array}\right)\left(\begin{array}{cc}
1 & -\zeta \\
0 & 1
\end{array}\right)=\left(\begin{array}{cc}
\cos \phi-\zeta \sin \phi & \left(1+\zeta^{2}\right) \sin \phi \\
-\sin \phi & \cos \phi+\zeta \sin \phi
\end{array}\right)
$$

which reproduces the matrices (2.15) for $\phi=\pi / 2$ and (2.19) for $\phi=-\pi / 2$.

Then, according to (2.16) and (2.17), we see that the fractional Appell transformation amounts to the replacement of the wavefunction $v(\xi, \zeta)$ by the wavefunction $w(\xi, \zeta)$ given by [9]

$$
w(\xi, \zeta)=\frac{e^{i \phi / 2}}{\sqrt{\cos \phi-\zeta \sin \phi}} e^{-i \frac{\xi^{2} \sin \phi}{2(\cos \phi-\zeta \sin \phi)}} v\left(\frac{\xi}{\cos \phi-\zeta \sin \phi}, \frac{\sin \phi+\zeta \cos \phi}{\cos \phi-\zeta \sin \phi}\right),
$$

as far as $\cos \phi-\zeta \sin \phi \neq 0$, whilst in the case when $\cos \phi-\zeta \sin \phi=0$ (i.e. $\zeta=\cot \phi$, which can occur for $\phi \in(-\pi,-\pi / 2] \cup[0, \pi / 2])$, it acts as

$$
w(\xi, \zeta)=\frac{e^{i(\phi-\pi / 2) / 2}}{\sqrt{\left(1+\zeta^{2}\right) \sin \phi}} e^{i \frac{\zeta}{1+\zeta^{2}} \xi^{2}} \widetilde{v}\left(\frac{\xi}{\left(1+\zeta^{2}\right) \sin \phi}, \zeta\right) \quad \text { for } \quad \zeta=\cot \phi
$$

The fractional Appell transformation identifies a family of transformations parameterized by the continuous parameter $\phi \in(-\pi, \pi]$. It would allow one to pass, for instance, from the $\psi_{\mathrm{KM}} \mathrm{S}$ to the $\psi_{\mathrm{BB}}$ through a continuum of "states" filling up the direct transformation $\psi_{\mathrm{KM}} \rightarrow \psi_{\mathrm{BB}}$, considered before, occurring in fact for $\phi=\pi / 2[9]$.

We conclude by recalling that, as a consequence of the properties of the fractional Fourier transform, the fractional Appell operator satisfies the basic properties:

(i) $\widehat{\mathcal{A}}^{\alpha}$ is continuous for all values of the order parameter $\alpha$,

(ii) $\widehat{\mathcal{A}}^{\alpha}$ obeys the group property, so that composing two operators of order $\alpha_{1}$ and $\alpha_{2}$ yields the operator of order $\alpha_{1}+\alpha_{2}$ :

$$
\widehat{\mathcal{A}}^{\alpha_{1}} \widehat{\mathcal{A}}^{\alpha_{2}}=\widehat{\mathcal{A}}^{\alpha_{1}+\alpha_{2}}=\widehat{\mathcal{A}}^{\alpha_{2}} \widehat{\mathcal{A}}^{\alpha_{1}},
$$

(iii) $\widehat{\mathcal{A}}^{\alpha}$ reduces to the ordinary operator for $\alpha=1: \widehat{\mathcal{A}}^{1}=\widehat{\mathcal{A}}$, and the identity operator for $\alpha=0: \widehat{\mathcal{A}}^{0}=\widehat{\mathrm{I}}$.

The property $(i)$ follows from the continuity of the Collins integral (2.10) on account of that of the ray-matrix entries as functions of $\phi$ (or, $\alpha$ ). Property (ii) ensues from the fact that the composition of the propagation integrals (2.10) reflects that of the ray-matrices, and hence $\widehat{\mathcal{A}}^{\alpha_{1}} \widehat{\mathcal{A}}^{\alpha_{2}} \leftrightarrow \mathbf{M}_{\widehat{\mathcal{A}}^{\alpha_{1}}} \mathbf{M}_{\widehat{\mathcal{A}}^{\alpha_{2}}}=\mathbf{M}_{\widehat{\mathcal{A}}^{\alpha_{1}+\alpha_{2}}} \leftrightarrow \widehat{\mathcal{A}}^{\alpha_{1}+\alpha_{2}}$ in accord with the addition formulae of the circular functions. Property $\left(\right.$ iii) is implied by that $\widehat{\mathcal{F}}^{1}=\widehat{\mathcal{F}}$ and $\widehat{\mathcal{F}}^{0}=\widehat{\mathrm{I}}$. In addition, as for $\widehat{\mathcal{F}}^{\alpha}$, the group property $(i i)$ allows $\widehat{\mathcal{A}}^{\alpha}$ to be mathematically understood as the $\alpha$-th power of $\widehat{\mathcal{A}}$.

Evidently, the expressions (2.21) and (2.26) conform to the well-known rule, which, according to standard results from Lie theory, conveys the action on functions in $\mathfrak{F}$ of any operator in the PWE symmetry group spanned by $\left\{\widehat{\mathrm{K}}_{+}, \widehat{\mathrm{K}}_{3}, \widehat{\mathrm{K}}_{-}\right\}[10,12]$. As earlier noted, the analysis above favors the "visualization" of the Appell transformation in optical terms, and makes also explicit the connection between some given transformations, which so appear as its "fractional powers".

\section{$2.22 \mathrm{D}$ "radial" paraxial wave equation}

As we know, the 3D PWE in (normalized) circular cylindrical coordinates $(\rho, \varphi, \zeta)$,

$$
\left[2 i \frac{\partial}{\partial \zeta}+\frac{\partial^{2}}{\partial \rho^{2}}+\frac{1}{\rho} \frac{\partial}{\partial \rho}+\frac{1}{\rho^{2}} \frac{\partial^{2}}{\partial \varphi^{2}}\right] u(\rho, \varphi, \zeta)=0
$$


with $\rho=\sqrt{\xi^{2}+\eta^{2}}$ and $\varphi=\arctan (\eta / \xi)$, allows for separable-variable solutions as

$$
u(\rho, \varphi, \zeta)=\Phi(\rho, \zeta) e^{i m \varphi}
$$

The evolution of the radial wavefunction $\Phi(\rho, \zeta)$ for a given azimuthal index $m$ is accordingly ruled by the $2 \mathrm{D}$ PWE in the radial coordinate $\rho$ :

$$
\left[2 i \frac{\partial}{\partial \zeta}+\frac{\partial^{2}}{\partial \rho^{2}}+\frac{1}{\rho} \frac{\partial}{\partial \rho}-\frac{m^{2}}{\rho^{2}}\right] \Phi(\rho, \zeta)=0
$$

In order to apply the above illustrated procedure to this equation, we resort to an appropriate representation of the $s l(2, \mathbb{R}) \simeq s u(1,1)$ generators for the symmetry of concern, namely

$$
\widehat{\mathcal{K}}_{+}:=\frac{1}{2} \rho^{2}, \quad \widehat{\mathcal{K}}_{-}:=-\frac{1}{2}\left(\frac{\partial^{2}}{\partial \rho^{2}}+\frac{1}{\rho} \frac{\partial}{\partial \rho}-\frac{m^{2}}{\rho^{2}}\right), \quad \widehat{\mathcal{K}}_{3}:=-\frac{i}{2}\left(\rho \frac{\partial}{\partial \rho}+1\right) .
$$

As their planar counterpart (2.4), the above relate to the operators $\widehat{\mathbf{x}}^{2}, \widehat{\mathbf{p}}^{2}$ and $\widehat{\mathbf{x}} \cdot \widehat{\mathbf{p}}$, properly expressed for a circular cylindrical symmetry.

The relevant propagator $\widehat{\mathcal{U}}_{\mathrm{PWE}}(\zeta):=\exp \left(-i \zeta \widehat{\mathcal{K}}_{-}\right)$amounts to a Hankel-like transform as that in rectangular coordinate amounts to the Fresnel transform (2.3). One has in fact:

$$
\Phi(\rho, \zeta)=\left[\widehat{\mathcal{U}}_{\mathrm{PWE}}(\zeta) f\right](\rho)=\frac{(-i)^{m+1}}{\zeta} \int_{0}^{\infty} e^{\frac{i}{2 \zeta}\left(\rho^{\prime 2}+\rho^{2}\right)} J_{m}\left(\frac{\rho \rho^{\prime}}{\zeta}\right) f\left(\rho^{\prime}\right) \rho^{\prime} d \rho^{\prime}
$$

$J_{m}$ denoting the Bessel function of the first kind and order $m$ [29]. Of course, $\Phi(\rho, 0)=f(\rho)$.

The above is a particular form of the (real or complex) radial canonical transform [21]

$$
\Phi\left(\rho, \zeta_{o}\right)=\widehat{\mathcal{U}}_{\left(\begin{array}{ll}
A & B \\
C & D
\end{array}\right)} \Phi\left(\rho, \zeta_{i}\right)=\frac{(-i)^{m+1}}{B} \int_{0}^{+\infty} e^{\frac{i}{2 B}\left(A \rho^{\prime 2}+D \rho^{2}\right)} J_{m}\left(\frac{\rho \rho^{\prime}}{B}\right) \Phi\left(\rho^{\prime}, \zeta_{i}\right) \rho^{\prime} d \rho^{\prime},
$$

corresponding to the canonical transformation conveyed by the (real or complex) symplectic matrix $\mathbf{M}=\left(\begin{array}{ll}A & B \\ C & D\end{array}\right)$. It basically follows from the $2 \mathrm{D}$ Collins integral under the circular cylindrical symmetry assumption (2.27). Hence, it relates the radial wavefunctions of the wavefields (of a given azimuthal symmetry) at the input and output planes at $\zeta_{i}$ and $\zeta_{o}$, between which the optical system, described by the ray-matrix $\mathbf{M}$, is conventionally intended to operate.

As (2.10), when $A \neq 0$ the diffraction integral (2.31) can be seen as resulting from a free propagation by $B / A$, followed by a lensing with focal power $-C$ and a scaling by $1 / A$, so that

$$
\Phi\left(\rho, \zeta_{o}\right)=\widehat{\mathcal{U}}_{\left(\begin{array}{ll}
A & B \\
C & D
\end{array}\right)} \Phi\left(\rho, \zeta_{i}\right)=\frac{1}{A} e^{i \frac{C}{2 A} \rho^{2}} \Phi\left(\frac{\rho}{A}, \zeta_{i}+\frac{B}{A}\right) .
$$

Here $\Phi\left(v, \zeta_{i}+B / A\right)=\left[\widehat{\mathcal{U}}_{\mathrm{PWE}}\left(\zeta_{i}+B / A\right) \Psi_{0}\right](v)$ represents the radial function composing the propagated form of the (effective or fictitious) source function $u(v, \varphi, 0)=\Psi_{0}(v) e^{i m \varphi}$, with which the wavefunction $u\left(v, \varphi, \zeta_{i}\right)=\Psi\left(v, \zeta_{i}\right) e^{i m \varphi}$ can be associated and whose azimuthal symmetry, accounted for by the factor $e^{i m \varphi}$ and supposedly preserved by the propagation, is implicitly conveyed by the dependence of the free-propagation integral (2.30) on the azimuthal index $m$.

In analogy with (2.17), the case $A=0$ yields the transformation

$$
\widehat{\mathcal{U}}_{\left(\begin{array}{ll}
0 & B \\
C & D
\end{array}\right)} \Psi\left(\rho, \zeta_{i}\right)=\frac{(-i)^{m+1}}{B} e^{\frac{i D}{2 B} \rho^{2}} \widetilde{\Psi}\left(\frac{\rho}{B}, \zeta_{i}\right)
$$

involving, as expected, the Hankel transform $\widetilde{\Psi}\left(\rho / B, \zeta_{i}\right)$ of order $m$ of the radial wavefunction at the input plane, modulated by the phase factor $\exp \left(i \frac{D}{2 B} \rho^{2}\right)$. 
As is well known, in fact, the $2 \mathrm{D}$ Fourier transform of a function $v(\rho, \varphi)$ obeying the factorization (2.27) with respect to the polar coordinates, i.e. $v(\rho, \varphi)=e^{i m \varphi} \Phi(\rho)$, turns into

$$
\widehat{\mathcal{F}}_{\xi} \widehat{\mathcal{F}}_{\eta} u(\rho, \varphi)=e^{i m(\varphi-\pi / 2)} \widetilde{\Phi}(\rho),
$$

with $\widetilde{\Phi}(\rho)$ signifying the Hankel transform of $\Phi(\rho)$ of order $m$, which according to the usual definition means

$$
\widetilde{\Phi}(\rho)=\left[\widehat{\mathcal{H}}_{m} \Phi\right](\rho):=\int_{0}^{\infty} J_{m}\left(\rho \rho^{\prime}\right) \Phi\left(\rho^{\prime}\right) \rho^{\prime} d \rho^{\prime} .
$$

As for (2.2), several solutions of (2.28) have been identified, which can be understood as arising from eigenstates of definite operators in the algebra generated by $\left\{\widehat{\mathcal{K}}_{+}, \widehat{\mathcal{K}}_{3}, \widehat{\mathcal{K}}_{-}\right\}[10,16]$. Thus, for instance, the eigenfunctions of the operator $\widehat{\mathcal{K}}_{\xi_{0}}=\widehat{\mathcal{K}}_{3}-\left(2 / \xi_{0}\right) \widehat{\mathcal{K}}_{+}$evolve into wavefunctions, which, as the aforementioned Weber-Hermite solutions of (2.2), depend on three independent parameters and comprise a complex quadratic exponential modulated by the Whittaker first function $M_{\kappa, \mu}$ of suitable argument [16]. As remarked, such solutions have been also deduced in [24] through an appropriate variable-separation ansatz.

\subsubsection{The Hankel transform and the optical (radial) Appell transformation}

In full analogy with the Fourier transform, the Hankel transform (2.34) of order $m$ can be given an operator representation in terms of the algebra generators $\widehat{\mathcal{K}}_{+}$and $\widehat{\mathcal{K}}_{-}$; namely,

$$
\widehat{\mathcal{H}}_{m}=i^{m+1} e^{-i(\pi / 2)\left[\widehat{\mathcal{K}}_{-}+\widehat{\mathcal{K}}_{+}\right]} .
$$

As before, the factor $i^{m+1}$ allows the mathematical transform (2.34) to be matched to the optical transform, conveyed by the Huygens-Hankel integral (2.31) for the Fourier matrix F.

Then, going through the same procedure as before, we can see that the radial Appell transformation (for a given azimuthal index $m$ ) is described by the operators

$$
\widehat{\mathcal{A}}_{m}:=e^{-i \zeta \widehat{\mathcal{K}}_{-}} \widehat{\mathcal{H}}_{m} e^{i \zeta \widehat{\mathcal{K}}_{-}} \quad \text { and } \quad \widehat{\mathcal{A}}_{m}^{-1}:=e^{-i \zeta \widehat{\mathcal{K}}_{-}} \widehat{\mathcal{H}}_{m}^{-1} e^{i \zeta \widehat{\mathcal{K}}_{-}}
$$

whose dependence on $\zeta$ will not explicitly displayed.

They respectively amount to the ABCD matrices (2.15) and (2.19), and hence, by (2.32), to the (radial) wavefunction transformations (for a given azimuthal index $m$ ):

$$
\Psi(\rho, \zeta)=\frac{( \pm i)^{m}}{i \zeta} e^{i \frac{\rho^{2}}{2 \zeta}} \Phi\left(\mp \frac{\rho}{\zeta},-\frac{1}{\zeta}\right)
$$

In accord with (2.33), accounting for the case $A=0$, we recover the Hankel transform-relation between the source functions: $\Psi(\rho, 0)=\widetilde{\Phi}(\rho, 0)$.

The two possibilities conveyed by (2.36) actually yield the same expression for the transformed wavefunction; this reflects the fact that $\widehat{\mathcal{A}}_{m}=\widehat{\mathcal{A}}_{m}^{-1}$ as a consequence of the self-reciprocity of the Hankel transform: $\widehat{\mathcal{H}}_{m}=\widehat{\mathcal{H}}_{m}^{-1}$.

In analogy with the "linear" operator $\widehat{\mathcal{A}}, \widehat{\mathcal{A}}_{m}$ can be regarded as a (back) evolving-location Hankel transform operator: $\widehat{\mathcal{A}}_{m}=\widehat{\mathcal{H}}_{m}(\zeta)$.

Paralleling the cases discussed in Section 2.1.2, we consider, as an Appell pair of solutions of (2.28), the Bessel beams [46] and the Bessel-Gauss beams [47]. The former can be interpreted as propagated forms of the eigenfunctions $J_{m}(\lambda \rho), \lambda \in \mathbb{R}$, of the free-Hamiltonian operator $\widehat{\mathcal{K}}_{-}$:

$$
B_{\lambda, m}(\rho, \zeta)=e^{-i \lambda^{2} \zeta / 2} J_{m}(\lambda \rho),
$$

the eigenvalue $\lambda$ signifying the transverse component of the wavenumber: $\lambda \leftrightarrow k_{\perp}[10,16]$. 
By the Appell transformation (2.36), the $B_{\lambda, m}$ s turn into the Bessel-Gauss beams:

$$
B G_{\lambda, m}(\rho, \zeta)=\frac{(-i)^{m+1}}{\zeta} e^{i \frac{\lambda^{2}}{2 \zeta}} e^{i \frac{\rho^{2}}{2 \zeta}} J_{m}\left(\lambda \frac{\rho}{\zeta}\right)
$$

which in turn arise from the eigenfunctions $\delta(\rho-\lambda), \lambda \in \mathbb{R}$, of the dual operator $\widehat{\mathcal{K}}_{+}[10,16]$.

As is well known, the Bessel modes are diffractionless (as the Airy beams $\psi_{\mathrm{BB}}{ }^{5}$ ), whilst the Bessel-Gauss modes, which as seen evolve from the Hankel transform of the source functions of the former, have complementary properties in both the space and spatial frequency domains.

Evidently, applying the radial fractional Appell transformation (for the given $m$ ), plainly understood as

$$
\widehat{\mathcal{A}}_{m}^{\alpha}:=e^{-i \zeta \widehat{\mathcal{K}}_{-}} \widehat{\mathcal{H}}_{m}^{\alpha} e^{i \zeta \widehat{\mathcal{K}}_{-}}=\widehat{\mathcal{H}}_{m}^{\alpha}(\zeta),
$$

one could follow the continuous transformation from the Bessel to the Bessel-Gauss modes at any $\zeta$. Here, $\widehat{\mathcal{H}}_{m}^{\alpha}$ signifies the fractional Hankel transform, which, resorting to the fractional Fourier transform matrix $\mathbf{F}^{\alpha}$, by (2.31) means

$$
\begin{aligned}
{\left[\widehat{\mathcal{H}}_{m}^{\alpha} \Phi\right](\rho) } & =\left[e^{i(m+1) \phi} e^{-i \phi\left[\widehat{\mathcal{K}}_{-}+\widehat{\mathcal{K}}_{+}\right]} \Phi\right](\rho) \\
& :=\frac{e^{i(m+1)(\phi-\pi / 2)}}{\sin \phi} \int_{0}^{\infty} e^{\frac{i \cos \phi}{2 \sin \phi}\left(\rho^{\prime 2}+\rho^{2}\right)} J_{m}\left(\frac{\rho \rho^{\prime}}{\sin \phi}\right) f\left(\rho^{\prime}\right) \rho^{\prime} d \rho^{\prime}
\end{aligned}
$$

with $\phi=\alpha \pi / 2 \in(-\pi, \pi]$. Therefore, as far as $\cos \phi-\zeta \sin \phi \neq 0, \widehat{\mathcal{A}}_{m}^{\alpha}$ yields the transformation

$$
\Psi(\rho, \zeta)=\frac{e^{i(m+1) \phi}}{\cos \phi-\zeta \sin \phi} e^{-i \frac{\rho^{2} \sin \phi}{2(\cos \phi-\zeta \sin \phi)} \Phi}\left(\frac{\rho}{\cos \phi-\zeta \sin \phi}, \frac{\sin \phi+\zeta \cos \phi}{\cos \phi-\zeta \sin \phi}\right),
$$

whilst for $\zeta=\cot \phi$ it acts as

$$
\Psi(\rho, \zeta)=\frac{e^{i(m+1)(\phi-\pi / 2)}}{\left(1+\zeta^{2}\right) \sin \phi} e^{i \frac{\zeta}{1+\zeta^{2}} \rho^{2}} \widetilde{\Phi}\left(\frac{\rho}{\left(1+\zeta^{2}\right) \sin \phi}, \zeta\right) \quad \text { for } \quad \zeta=\cot \phi
$$

\subsection{Optical Appell transformation and duality. Self-Fourier/self-Hankel and self-Appell wavefunctions}

Since, as reviewed above, the Appell transformation manifests the correspondence between wavefunctions generated by Fourier or Hankel pairs of functions [9], it naturally relates to the concept of beam duality [48, 49]. However, it does not connect wavefunctions, which are locally dual, but connects wavefunctions whose duality traces back to the respective source functions.

An "Appell transformer" - provided it be implementable - would be so an optical device turning a wavefunction into that which one would generate by propagating the Fourier transform of the source function of the original wavefunction. In a sense, as the Fourier transformer can be understood as a "local dual switch", the "Appell transformer" might be understood as an "initial-plane dual switch". Correspondingly, the fractional Appell transformations individualize a family of symmetry transformations parameterized by a continuous parameter, which, filling the gap between the "evolution-lines" of a function and its Fourier/Hankel transform, might be seen as relating to a sort of "fractional beam duality".

In the light of the examples considered above, i.e. Airy and Bessel beams, we may visualize the problem in terms of two ideal paths, running parallel to each other to depict the $\zeta$-lines along which the evolution of a given source function and of its Fourier or Hankel transform occur. The Appell transformation connects one path to the other at any desired $\zeta$ or better one path to any

\footnotetext{
${ }^{5}$ Note that the comparison should more correctly involve the eigenfunctions of $\widehat{\mathrm{P}}$, i.e. the plane waves $e^{i \lambda \xi}$.
} 
other path between the two "extreme" ones, thus allowing us to "have a look" at or to turn the wavefunction at hand into a wavefunction whose properties in the space and spatial frequency domains are a desired mixture of those of the wavefunctions "lying" on the two extreme paths.

Evidently, when the source functions are self-Fourier or self-Hankel functions, the two paths collapse one into the other. The Appell transformation in fact comes to reproduce at any $\zeta$ the direct (or, inverse) self-Fourier/self-Hankel relations obeyed by the source functions. This is the case, for instance, of the standard Hermite-Gauss and Laguerre-Gauss modes, which respectively arise from the eigenfunctions of the self-dual Lie operators $\widehat{\mathrm{K}}_{-}+\widehat{\mathrm{K}}_{+}$and $\widehat{\mathcal{K}}_{-}+\widehat{\mathcal{K}}_{+}$; as seen above, the latter are the generators of the (both ordinary and fractional) Fourier and Hankel transforms.

In fact, taking into account the explicit expressions for the quoted (normalized) modes, i.e.

$$
s H G_{n}(\xi, \zeta)=\frac{1}{\sqrt{2^{n} n ! \mu(\zeta) \sqrt{\pi}}}\left(\frac{\mu^{*}(\zeta)}{\mu(\zeta)}\right)^{n / 2} e^{-\frac{\xi^{2}}{2 \mu(\zeta)}} H_{n}\left(\frac{\xi}{|\mu(\zeta)|}\right),
$$

for the standard Hermite-Gauss modes, and

$$
s L G_{n, m}(\rho, \zeta)=\sqrt{\frac{2 n !}{(n+m) !}} \frac{1}{\mu(\zeta)^{m+1}}\left(\frac{\mu^{*}(\zeta)}{\mu(\zeta)}\right)^{n} \rho^{m} e^{-\frac{\rho^{2}}{2 \mu(\zeta)}} L_{n}^{m}\left(\frac{\rho^{2}}{|\mu(\zeta)|^{2}}\right),
$$

for the standard Laguerre-Gauss modes, where

$$
\mu(\zeta)=1+i \zeta
$$

$H_{n}$ denotes the Hermite polynomial of degree $n$ and $L_{n}^{m}$ the generalized Laguerre polynomial of degree $n$ and order $m$ [29], we can verify that

$$
\begin{aligned}
& \widehat{\mathcal{A}}^{\alpha} s H G_{n}(\xi, \zeta)=(-i)^{\alpha n} s H G_{n}(\xi, \zeta), \\
& \widehat{\mathcal{A}}_{m}^{\alpha} s L G_{n, m}(\rho, \zeta)=(-1)^{\alpha n} s L G_{n, m}(\rho, \zeta) .
\end{aligned}
$$

The above just reproduce the relations holding between the relevant source functions, which are the Hermite-Gauss and Laguerre-Gauss functions $s H G_{n}(\xi, 0)=\frac{1}{\sqrt{2^{n} n ! \sqrt{\pi}}} e^{-\xi^{2} / 2} H_{n}(\xi)$ and $s L G_{n, m}(\rho, 0)=\sqrt{\frac{2 n !}{(n+m) !}} \rho^{m} e^{-\rho^{2} / 2} L_{n}^{m}\left(\rho^{2}\right)$, and the respective Fourier and Hankel transforms.

In a sense, the standard Hermite-Gauss and Laguerre-Gauss modes can be considered as selfAppell wavefunctions, respectively suitable to a rectangular and circular cylindrical geometry.

\section{Canonical transforms: a short review}

As seen, the interpretation of the Appell transformation within the optical context naturally resorts to the Fourier and Hankel transforms [9], which ultimately are integral transforms.

In general, integral transforms provide a well established and valuable method to solve problems in several areas of both physics and applied mathematics. As we know, the roots of the method can be traced back to the original work by Oliver Heaviside on the ordinary differential equations with constant coefficients occurring in the theory of electric circuits [50,51]. Due also to the availability of large scale computers, the method has then been increasingly extended to a wide range of physical and mathematical problems, basically described by partial differential equations with assigned boundary and initial conditions [52, 53, 13].

In particular, a special class of integral transforms, named canonical transforms (CT), appear widely in optics, in electromagnetism, in classical and quantum mechanics as well as in computational and applied mathematics. Their link to the canonical transformations and to 
the parabolic differential equations as well as to the theory of special functions has been deeply analyzed. Fourier, bilateral Laplace, Bargmann, Weierstrass-Gauss transforms as well as Hankel and Barut-Girardello transforms are examples of CTs.

Canonical transformations play a crucial role in classical mechanics. When applying the inherent formalism to quantum mechanics, the CTs naturally arise (under specific conditions) as associated representations (unitary or not) acting between suitably constructed Hilbert spaces of functions on the real or complex domain [54, 55, 56, 57, 58, 59].

On the other hand, the initial value problem for evolutionary equations is usually formulated in terms of the evolution (or, displacement) operator. The 2D PWE and the $(1+1) \mathrm{D}$ SE are examples of evolution equations, whose displacement operators realize a unitary mapping of the Hilbert space of square integrable functions into itself. The same can be said for the 1D HE although in this case the relevant evolution operator no longer generates a unitary mapping. It is proved that CTs can be realized as evolution operators, generated by second-order differential operators through exponentiation to the group by real or complex parameters, and hence as such can be associated with evolution equations, ruled by Hamiltonian-like operators (not necessarily Hermitian) which are quadratic in the inherent canonically conjugate variables [60].

In addition, CTs directly relate to some aspects of the theory of special functions through the eigenvalue problem [61]. In fact, special functions, like the Hermite-Gauss, the Laguerre-Gauss and the parabolic cylinder functions, are self-reciprocal under some of the aforementioned CTs.

The Fourier and Hankel transforms are basic examples of CTs. Their role has been enlarged to more general contexts by the introduction of the corresponding transforms of fractional order [62, $63,64]$, which, for instance, in the field of optics [42, 43, 44, 45], gave rise to a great variety of applications, investigations and new formulations in an increasingly enriched optics scenario, that in turn stimulated further general analyses of the linear CTs from both theoretical and applicative/numerical points of view. Few recent titles are in [65, 66, 67, 68, 69, 70].

The Fourier and Hankel transforms are real (respectively, linear and radial) CTs. In Section 4.2 we will see that the caloric Appell transformation, relevant to the 1D HE, naturally relates to the bilateral Laplace transform, which in contrast is a special type of complex (linear) CTs.

\subsection{Linear canonical transforms}

We recall that real linear ${ }^{6}$ CTs realize a unitary mapping of the Hilbert space $\mathfrak{L}^{2}(\mathbb{R})$ into itself through the integral transform $[55,56,57,58]$

$$
\widehat{\mathrm{T}}_{\mathrm{M}}: \varphi \in \mathfrak{L}^{2}(\mathbb{R}) \rightarrow\left[\widehat{\mathrm{T}}_{\mathrm{M}} \varphi\right](x):=\int_{-\infty}^{\infty} \mathrm{K}_{\mathrm{M}}\left(x, x^{\prime}\right) \varphi\left(x^{\prime}\right) d x^{\prime} \in \mathfrak{L}^{2}(\mathbb{R}),
$$

whose kernel

$$
\mathrm{K}_{\mathrm{M}}\left(x, x^{\prime}\right):=\frac{1}{\sqrt{2 \pi i B}} e^{\frac{i}{2 B}\left(A x^{2}+D x^{2}-2 x x^{\prime}\right)}
$$

depends on the three linearly independent entries $A, B, C$ of a $2 \times 2$ unimodular real matrix $\mathbf{M}=\left(\begin{array}{ll}A & B \\ C & D\end{array}\right)$, with the aforestated convention on the square root of a (complex) number and the requirement $A / B \geq 0$ resulting form the integrability condition. The matrix $\mathbf{M}$ belongs to the three-parameter real symplectic group $S p(2, \mathbb{R}) \simeq S L(2, \mathbb{R})$, and in practice specifies the canonical transformation of the involved (classical or quantum) canonically conjugate variables ${ }^{7}$.

\footnotetext{
${ }^{6}$ The term linear accounts for the integration involving the real line $\mathbb{R}$.

${ }^{7}$ As is well known, classically a canonical transformation is a change of the phase-space variables $(q, p) \rightarrow$ $\left(q^{\prime}(q, p), p^{\prime}(q, p)\right)$, which preserves the Poisson bracket $\{q, p\}=1=\left\{q^{\prime}, p^{\prime}\right\}$. As a straightforward extension of the above concept, a quantum canonical transformation is defined as a change of the conjugate non-commuting observables $\widehat{q} \rightarrow \widehat{q}^{\prime}(\widehat{q}, \widehat{p}), \widehat{p} \rightarrow \widehat{p}^{\prime}(\widehat{q}, \widehat{p})$, which preserves the Dirac bracket $[\widehat{q}, \widehat{p}]=i=\left[\widehat{q}^{\prime}, \widehat{p}^{\prime}\right]$.
} 
Notably, the inverse transform is given by the same expression with $\mathbf{M}$ replaced by the inverse $\mathbf{M}^{-1}: \widehat{\mathbf{T}}_{\mathbf{M}}^{-1}=\widehat{\mathrm{T}}_{\mathbf{M}^{-1}}$, thus amounting to the kernel $\mathrm{K}_{\mathbf{M}^{-1}}\left(x, x^{\prime}\right)=\mathrm{K}_{\mathbf{M}}^{*}\left(x^{\prime}, x\right)$.

As said, the Collins integral (2.10) is a real linear CT, the relevant matrix $\mathbf{M}$ signifying in fact the transformation of the paraxial-ray variables consequent to the propagation through the optical system described by $\mathbf{M}$.

The case $B=0$, which optically signifies imaging, yields the geometric transform

$$
\widehat{\mathrm{T}}_{\left(\begin{array}{ll}
A & 0 \\
C & D
\end{array}\right)}: \varphi \in \mathfrak{L}^{2}(\mathbb{R}) \rightarrow \frac{1}{\sqrt{A}} e^{i \frac{C}{2 A} x^{2}} \varphi\left(\frac{x}{A}\right) \in \mathfrak{L}^{2}(\mathbb{R}) .
$$

The extension of real CTs to complex CTs is rather involved. For an accurate account, we address the reader to the devoted literature $[54,55,56,57,58,59]$. Here, we briefly recall that complex linear CTs amount to the same integral transform (3.1), (3.2), but the kernel involves the three linearly independent entries of a $2 \times 2$ unimodular complex matrix $\mathbf{M}$, belonging to the six-parameter complex symplectic group $S p(2, \mathbb{C}) \simeq S L(2, \mathbb{C})$; accordingly, the integrability condition requires now that $\Im(A / B) \geq 0$ or $B$ real if $A=0$. In fact, when extending the concept of canonical transformation from classical to quantum mechanics, it seemed useful to extend the transformation from the real to the complex domain. This implies that Hermitian operators can be mapped into canonically conjugate, but not necessarily Hermitian, operators. Then, contrary to the real CTs, complex CTs no longer represent unitary mappings from $\mathfrak{L}^{2}(\mathbb{R})$ to $\mathfrak{L}^{2}(\mathbb{R})$; they transfer $\mathfrak{L}^{2}(\mathbb{R})$ into the Bargmann-Hilbert space $\mathfrak{F}_{\mathrm{M}}$ of analytic square-integrable functions over the complex plane [54], completed by a suitably defined scalar product in order that the transformed operators have the appropriate hermiticity properties and reproduce in the new variable the Schrödinger representation as $x$ and $-i d / d x$. Specifically, the complex $\mathrm{CT} \widehat{\mathrm{T}}_{\mathbf{M}}$ associated with the transformation matrix $\mathbf{M} \in S p(2, \mathbb{C})$ amounts to the transform pair [57]

$$
\begin{aligned}
& \widetilde{\varphi}(x)=\left[\widehat{\mathrm{T}}_{\mathrm{M}} \varphi\right](x)=\int_{\mathbb{R}} d x^{\prime} \mathrm{K}_{\mathrm{M}}\left(x, x^{\prime}\right) \varphi\left(x^{\prime}\right), \\
& \varphi\left(x^{\prime}\right)=\int_{\mathbb{C}} d \mu_{\mathrm{M}}(x) \mathrm{K}_{\mathrm{M}}^{*}\left(x, x^{\prime}\right) \widetilde{\varphi}(x),
\end{aligned}
$$

connecting $\mathfrak{L}^{2}(\mathbb{R})$ and $\mathfrak{F}_{\mathrm{M}}$, where

$$
(\widetilde{\varphi}, \widetilde{\psi})_{\widetilde{F}_{\mathbf{M}}}=\int_{\mathbb{C}} d \mu_{\mathbf{M}}(x) \widetilde{\varphi}^{*}(x) \widetilde{\psi}(x),
$$

the measure being

$$
d \mu_{\mathbf{M}}(x)=\sqrt{\frac{2}{\pi v}} e^{\frac{1}{2 v}\left(u x^{2}-2 x x^{*}+u^{*} x^{* 2}\right)} d \Re x d \Im x,
$$

with $u=A^{*} D-B^{*} C \in \mathbb{C}$ and $v=2 \Im\left(B^{*} A\right) \in \mathbb{R}[54,55,56,57,58,59]$.

A basic example of complex linear CT is provided by the Bargmann transform [54]

$$
[\widehat{\mathbb{B}} \varphi](x):=\frac{1}{\pi^{1 / 4}} \int_{-\infty}^{+\infty} d x^{\prime} e^{-\left(x^{\prime 2}+x^{2}-2 \sqrt{2} x x^{\prime}\right) / 2} \varphi\left(x^{\prime}\right)=(2 \pi)^{1 / 4}\left[\widehat{\mathbf{T}}_{\mathbf{B}} \varphi\right](x),
$$

the relevant canonical transformation, signified by the Bargmann matrix

$$
\mathbf{B}=\frac{1}{\sqrt{2}}\left(\begin{array}{cc}
1 & -i \\
-i & 1
\end{array}\right)
$$

essentially turning the coordinate and momentum operators into the harmonic oscillator raising and lowering operators. Accordingly, the Bargmann transform relates the Schrödinger and Fock 
representations of quantum mechanics; it is in fact applied, for instance, to the coherent-state formulation of quantum optics. Evidently, $\mathfrak{F}_{\mathrm{B}}$ is the space of holomorphic functions on $\mathbb{C}$ which are square integrable with respect to a Gaussian measure.

We will specifically deal below with two kinds of complex linear CTs, associated with the transformation matrices

$$
\mathbf{P}(\tau)=\left(\begin{array}{cc}
1 & -i \tau \\
0 & 1
\end{array}\right), \quad \tau>0
$$

and

$$
\mathbf{G}(1 / w)=\left(\begin{array}{cc}
1 & 0 \\
i / w & 1
\end{array}\right), \quad w>0
$$

The former produces the Poisson (or, Weierstrass-Gauss) transform

$$
\left[\widehat{\mathrm{T}}_{\mathbf{P}} \varphi\right](x):=\frac{1}{\sqrt{2 \pi \tau}} \int_{-\infty}^{+\infty} e^{-\frac{1}{2 \tau}\left(x-x^{\prime}\right)^{2}} \varphi\left(x^{\prime}\right) d x^{\prime}
$$

which may account, for instance, for heat conduction (Section 4.1). It may be considered as the complex counterpart of the Fresnel transform (2.3) accounting for paraxial free-propagation, the relevant transformation matrix being (2.11). Similarly, it transfers the initial function $\varphi(x)$ at $\tau=0$ into the temperature function $\psi(x, \tau)=\left[\widehat{T}_{\mathbf{P}} \varphi\right](x)$ at subsequent $\tau \mathrm{s}$. Interestingly, since the Poisson transform formally implies the convolution with a pure Gaussian function, it is optically implementable by a Fourier transform followed by the propagation through a Gaussian aperture followed in turn by an inverse Fourier transform. The parameter $1 / \tau$ takes the meaning as the characteristic width of the involved Gaussian function.

As to (3.7), we see that according to (3.3) it amounts to a modulation by the Gaussian $\exp \left(-x^{2} / 2 w\right)$, which is optically realizable by propagation through a Gaussian aperture, the parameter $w$ signifying then the characteristic width of the aperture. Evidently, the real matrix

$\mathbf{L}(1 / f)=\left(\begin{array}{cc}1 & 0 \\ -1 / f & 1\end{array}\right)$, amounting to the multiplication by the phase factor $\exp \left(-i x^{2} / 2 f\right)$, is the real counterpart of (3.7); it signifies indeed the propagation through a thin lens.

As their real counterparts, the above matrices obey the semigroup property: $\mathbf{P}\left(\tau_{1}\right) \mathbf{P}\left(\tau_{2}\right)=$ $\mathbf{P}\left(\tau_{2}\right) \mathbf{P}\left(\tau_{1}\right)=\mathbf{P}\left(\tau_{1}+\tau_{2}\right)$ and $\mathbf{G}\left(1 / w_{1}\right) \mathbf{G}\left(1 / w_{2}\right)=\mathbf{G}\left(1 / w_{2}\right) \mathbf{G}\left(1 / w_{1}\right)=\mathbf{G}\left(1 / w_{1}+1 / w_{2}\right)$, and represent a dual pair of operators, since $\mathbf{P}(\tau)=\mathbf{F}^{-1} \mathbf{G}(\tau) \mathbf{F}$ and similarly $\mathbf{G}(1 / w)=\mathbf{F}^{-1} \mathbf{P}(1 / w) \mathbf{F}$.

An interesting pair of related real/complex CTs is represented by the Fourier and bilateral Laplace transform, whose features of interest here will be reviewed below.

\subsubsection{The bilateral Laplace transform}

The bilateral Laplace transform $\widehat{\mathbb{L}}$ is well known to transfer an in general complex-valued function defined over $\mathbb{R}$ into a complex-valued function defined over $\mathbb{C}$ by the integral

$$
[\widehat{\mathbb{L}} \varphi](x):=\int_{-\infty}^{+\infty} d x^{\prime} e^{-x x^{\prime}} \varphi\left(x^{\prime}\right)
$$

simply referred to as Laplace transform in the following.

It can be recovered within the formalism of the complex linear CTs. In fact, according to (3.4), the unimodular complex matrix

$$
\mathbf{L}=\left(\begin{array}{ll}
0 & i \\
i & 0
\end{array}\right)
$$


yields the integral transform $\widehat{T}_{\mathbf{L}}$, which is off of $\widehat{\mathbb{L}}$ by the factor $-i / \sqrt{2 \pi}$, being

$$
\left[\widehat{\mathbf{T}}_{\mathbf{L}} \varphi\right](x)=\frac{1}{i \sqrt{2 \pi}} \int_{-\infty}^{+\infty} d x^{\prime} e^{-x x^{\prime}} \varphi\left(x^{\prime}\right)=\frac{1}{i \sqrt{2 \pi}}[\widehat{\mathbb{L}} \varphi](x)
$$

As noted in [57], since $\mathbf{L}$ does not fullfil the aforestated integrability condition that $B$ be real if $A=0$, the relevant transform might be meaningless as the inherent integral might be divergent. Indeed, we know that a critical issue in dealing with Laplace transform is convergence, since $[\widehat{\mathbb{L}} \varphi](x)$ generally exists only for some values of $x$, located in the region of convergence (determined by $\varphi$ and $\Re x$ ). However, we will not dwell here on such a question, since we will not go through the evaluation of the Laplace transform of specific functions, but we will exploit its interpretation as a linear CT with the associated transformation matrix (3.9) and the consequent exponential operator representation in terms of the $s u(1,1)$-generators $\widehat{\mathrm{K}}_{-}$and $\widehat{\mathrm{K}}_{+}$. In fact, in analogy with the Fourier transform (see (2.9)), $\widehat{T}_{\mathbf{L}}$ can be expressed in the form

$$
\widehat{\mathrm{T}}_{\mathbf{L}}=e^{(\pi / 2)\left[\widehat{\mathrm{K}}_{-}-\widehat{\mathrm{K}}_{+}\right]},
$$

and so interpreted as the non-unitary evolution operator describing the dynamics of a quantum repulsive oscillator observed at the purely imaginary "time" $\tau=i \pi / 2[60,61]$, whereas, as earlier noted, the Fourier transform can be understood as the unitary evolution operator describing the dynamics of the quantum attractive oscillator observed at the real "time" $\tau=\pi / 2$. In this connection, it may be worth recalling that the operators $\widehat{\mathrm{K}}_{-}+\widehat{\mathrm{K}}_{+} \propto \widehat{\mathrm{P}}^{2}+\widehat{\mathrm{X}}^{2}$ and $\widehat{\mathrm{K}}_{-}-\widehat{\mathrm{K}}_{+} \propto$ $\widehat{\mathrm{P}}^{2}-\widehat{\mathrm{X}}^{2}$ span by exponentiation the elliptic and hyperbolic subgroups of the symplectic group.

Moreover, paralleling the factorizations $(2.13)$ of $\widehat{\mathcal{F}}$, we similarly write

$$
\widehat{\mathrm{T}}_{\mathbf{L}}=e^{\widehat{\mathrm{K}}_{-}} e^{-\widehat{\mathrm{K}}_{+}} e^{\widehat{\mathrm{K}}_{-}}=e^{-\widehat{\mathrm{K}}_{+}} e^{\widehat{\mathrm{K}}_{-}} e^{-\widehat{\mathrm{K}}_{+}},
$$

since, along with (2.12), one also has

$$
\begin{aligned}
e^{\beta\left[\widehat{\mathrm{K}}_{-}-\widehat{\mathrm{K}}_{+}\right]} & =e^{\tan (\beta / 2) \widehat{\mathrm{K}}_{-}} e^{-\sin (\beta) \widehat{\mathrm{K}}_{+}} e^{\tan (\beta / 2) \widehat{\mathrm{K}}_{-}} \\
& =e^{-\tan (\beta / 2) \widehat{\mathrm{K}}_{+}} e^{\sin (\beta) \widehat{\mathrm{K}}_{-}} e^{-\tan (\beta / 2) \widehat{\mathrm{K}}_{+}}, \quad|\beta|<\pi .
\end{aligned}
$$

As is well known, the Fourier transform admits as eigenfunctions the Hermite-Gauss functions,

$$
u_{n}(x)=\frac{1}{\sqrt{2^{n} n ! \sqrt{\pi}}} e^{-x^{2} / 2} H_{n}(x)
$$

(that in Section 2.3 have been seen to be the source functions $s H G(\xi, 0)$ for the standard Hermite-Gauss modes), being in fact

$$
\widehat{\mathcal{F}} u_{n}=(-i)^{n} u_{n}
$$

In contrast, the eigenfunctions of $\widehat{T}_{\mathbf{L}}$ involve the Weber-Hermite functions $D_{\nu}$, which for integer orders turn into the $u_{n} \mathrm{~s}: D_{n}(\sqrt{2} x)=\sqrt{n ! \sqrt{\pi}} u_{n}(x)$ [29]. The eigenvalue equation for $\widehat{\mathrm{T}}_{\mathbf{L}}$,

$$
\widehat{\mathrm{T}}_{\mathbf{L}} \Phi_{\mu}^{ \pm}=\lambda_{\mathbf{L}}(\mu) \Phi_{\mu}^{ \pm}
$$

is in fact solved by

$$
\Phi_{\mu}^{ \pm}(x)=\frac{\Gamma(-i \mu+1 / 2)}{2^{3 / 4} \pi} e^{-i \pi(i \mu+1 / 2) / 4} D_{i \mu-1 / 2}\left( \pm e^{i 3 \pi / 4} \sqrt{2} x\right), \quad \lambda_{\mathbf{L}}(\mu)=e^{\pi \mu / 2},
$$


for real values of $\mu[10,60,61]$. The functions $\Phi_{\mu}^{ \pm}$s provide an eigenfunction basis for $\mathfrak{L}^{2}(\mathbb{R})$ as well as for the Bargmann-Hilbert space $\mathfrak{F}_{\mathbf{L}}$.

In [66] a possible definition of fractional Laplace transform has been proposed by applying to the fractional Fourier transform matrix $\mathbf{F}^{\alpha}$ the same similarity transformation which through the ordinary Fourier transform matrix $\mathbf{F}$ yields $\mathbf{L}$. In fact, since ${ }^{8}$

$$
\mathbf{L}=\mathbf{S}\left(e^{i \pi / 4}\right) \mathbf{F S}\left(e^{-i \pi / 4}\right)
$$

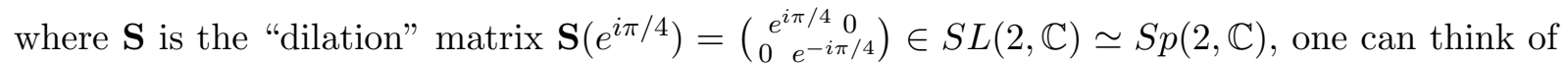
reproducing the above scheme with $\mathbf{F}^{\alpha}$ in place of $\mathbf{F}$. The resulting matrix

$$
\mathbf{L}^{\alpha}=\mathbf{S}\left(e^{i \pi / 4}\right) \mathbf{F}^{\alpha} \mathbf{S}\left(e^{-i \pi / 4}\right)=\left(\begin{array}{cc}
\cos \phi & i \sin \phi \\
i \sin \phi & \cos \phi
\end{array}\right), \quad \phi=\alpha \pi / 2
$$

has been taken in [66] as the representative matrix of the fractional Laplace transform $\widehat{\mathcal{L}}^{\alpha}$ of order $\alpha$ by the definition

$$
\widehat{\mathcal{L}}^{\alpha}=i^{\alpha / 2} \widehat{\mathbf{T}}_{\mathbf{L}^{\alpha}} .
$$

Then, echoing the fractional Fourier transform (see (2.24)), $\widehat{\mathcal{L}}^{\alpha}$ amounts to the integral transform

$$
\left[\widehat{\mathcal{L}}^{\alpha} \varphi\right](x)=\sqrt{\frac{1-i \cot \phi}{2 \pi i}} \int_{-\infty}^{+\infty} d x^{\prime} e^{\frac{1}{2 \sin \phi}\left(x^{\prime 2} \cos \phi+x^{2} \cos \phi-2 x x^{\prime}\right)} \varphi\left(x^{\prime}\right)
$$

The ordinary transform is recovered with $\alpha=1$, which yields $\widehat{\mathcal{L}}^{1}=i^{1 / 2} \widehat{\mathrm{T}}_{\mathbf{L}}=(2 \pi i)^{-1 / 2} \widehat{\mathbb{L}}$.

In analogy with $\widehat{\mathcal{F}}^{\alpha}$, one can prove for $\widehat{\mathcal{L}}^{\alpha}$ the relations

$$
\begin{aligned}
& \widehat{\mathcal{L}}^{\alpha+4 j}=\widehat{\mathcal{L}}^{\alpha}, \quad j=0, \pm 1, \pm 2, \ldots, \\
& \widehat{\mathcal{L}}^{\alpha}=(\widehat{\mathcal{L}})^{\alpha},
\end{aligned}
$$

the periodicity with respect to the order $\alpha$ allowing us to limit it to the interval $(-2,2]$, whereas the power-like relation implies that $\widehat{\mathcal{L}}^{\alpha}$ admits the same eigenfunctions $\Phi_{\mu}^{ \pm}$as $\widehat{\mathcal{L}}$ (and so $\widehat{T}_{\mathbf{L}}$ ):

$$
\widehat{\mathcal{L}}^{\alpha} \Phi_{\mu}^{ \pm}=\lambda_{\mathcal{L}^{\alpha}}(\mu) \Phi_{\mu}^{ \pm}, \quad \lambda_{\mathcal{L}^{\alpha}}(\mu)=i^{\alpha / 2}\left[\lambda_{\mathbf{L}}(\mu)\right]^{\alpha}=i^{\alpha / 2} e^{\pi \alpha \mu / 2}
$$

It has recently been noted [71] that the transform $\widehat{\mathcal{L}}^{\alpha}$, as proposed in [66] through (3.13), does not reproduce the fractional Fourier transform (2.24) when the real part of the transform variable $x^{\prime}=\Re x^{\prime}+i \Im x^{\prime}$ is set to zero. Then, in order to overcome such a limit, a different parameter matrix, precisely $\mathbf{L}^{\prime \alpha}=\left(\begin{array}{cc}i \cos \phi & i \sin \phi \\ i \sin \phi & -i \cos \phi\end{array}\right)$, has been suggested in the quoted reference to define the fractional Laplace transform through the usual scheme (3.4) pertaining to the linear CTs. We have mentioned such a result for completeness' sake, but we will not use the proposed matrix $\mathbf{L}^{\prime \alpha}$ in the forthcoming analysis. Actually, also $\mathbf{L}^{\alpha}$ will marginally be used below.

\footnotetext{
${ }^{8}$ It may be worth noting that it is also $\mathbf{L}=\mathbf{S}\left(e^{i \pi / 2}\right) \mathbf{F}=\mathbf{F S}\left(e^{-i \pi / 2}\right)$, and hence $\widehat{T}_{\mathbf{L}}$ relates to $\widehat{T}_{\mathbf{F}}$ with the relevant integral involving the imaginary axis. As remarked in [57], in fact, multiplication of the transformation matrix $\mathbf{M}$ on the left (on the right) by the "dilation" matrix $\mathbf{S}\left(e^{i \alpha}\right)\left(\mathbf{S}\left(e^{-i \alpha}\right)\right)$ is a tool to obtain transforms involving line integrals along a path tilted by the phase $\alpha$.
} 


\subsubsection{Some about the Laplace transform representative matrix}

The representative matrix of the Laplace transform, be it $\mathbf{L}$ or $\mathbf{L}^{\alpha}$, if we are dealing with the fractional transform (3.13), belongs to the set of complex unimodular matrices as

$$
\mathbf{M}=\left(\begin{array}{cc}
A & i B \\
-i C & D
\end{array}\right)
$$

with $A, B, C, D$ real, and $A D-B C=1$, which indeed form a subgroup since the product of two matrices of this type yields a matrix of the same form.

Complex canonical transformations, conveyed by matrices like (3.14), and their nonunitary representations have a number of interesting applications in physics. They arise, for instance, in the clustering theory of nuclei [59] as well as in the study of the accidental degeneracy [72] in both a 2D anisotropic oscillator, whose frequencies in the two directions have a rational ratio, and a $2 \mathrm{D}$ isotropic oscillator, constrained to move in a sector of angle $\pi / q, q$ integer.

Also, the matrices (3.6) and (3.7), representing optical processes like the convolution by a Gaussian function and the propagation through a Gaussian aperture, are of the type (3.14).

Specifically, the matrices (3.14) produce the integral transform

$$
\left[\widehat{T}_{\left(\begin{array}{c}
A \\
-i C
\end{array} \underset{D}{i B}\right)} \varphi\right](x)=\frac{1}{\sqrt{-2 \pi B}} \int_{-\infty}^{+\infty} e^{\frac{1}{2 B}\left(A x^{2}+D x^{2}-2 x x^{\prime}\right)} \varphi\left(x^{\prime}\right) d x^{\prime} .
$$

As the Huygens-Fresnel transform (2.10), it can be recast in a form similar to (2.16). In fact, (2.16) reflects the factorization $\grave{a}$ la Wei-Norman of real symplectic matrices [28],

$$
\left(\begin{array}{ll}
A & B \\
C & D
\end{array}\right)=\left(\begin{array}{cc}
1 & 0 \\
C / A & 1
\end{array}\right)\left(\begin{array}{cc}
A & 0 \\
0 & 1 / A
\end{array}\right)\left(\begin{array}{cc}
1 & B / A \\
0 & 1
\end{array}\right),
$$

in terms of a dilation matrix sandwiched between two real triangular matrices, respectively lower-left and upper-right, which optically account for lensing and free-propagation.

A similar factorization of matrices of the type (3.14) is allowed as

$$
\left(\begin{array}{cc}
A & i B \\
-i C & D
\end{array}\right)=\left(\begin{array}{cc}
1 & 0 \\
i / w & 1
\end{array}\right)\left(\begin{array}{cc}
A & 0 \\
0 & 1 / A
\end{array}\right)\left(\begin{array}{cc}
1 & -i \tau \\
0 & 1
\end{array}\right)
$$

with the real parameters $\tau$ and $w$ given by

$$
\tau=-\frac{B}{A}, \quad \frac{1}{w}=-\frac{C}{A} .
$$

Thus, by (3.16) the transform (3.15) can alternatively be understood as

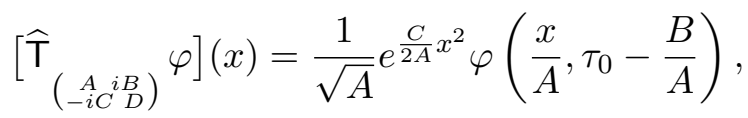

where $\varphi\left(x, \tau_{0}-B / A\right)$ is to be intended as the "Poisson"-transformed (i.e. in a sense, "evolved" with respect to the effective or fictitious parameter $\tau_{0}$ ) form of the function $\varphi(x)$, namely

$$
\varphi\left(x, \tau_{0}+\tau\right)=\frac{1}{\sqrt{2 \pi \tau}} \int_{-\infty}^{+\infty} e^{-\frac{1}{2 \tau}\left(x-x^{\prime}\right)^{2}} \varphi_{0}\left(x^{\prime}\right) d x^{\prime}=\left[\widehat{\mathbf{T}}_{\mathbf{P}} \varphi_{0}\right](x) .
$$

with $\varphi_{0}(x)=\varphi\left(x, \tau_{0}\right)$.

As for real matrices, the factorization (3.16) holds for $A \neq 0$. If $A=0$, the general expression (3.15) yields

$$
\left[\widehat{\mathrm{T}}_{\left(\begin{array}{c}
0 \\
-i C
\end{array} \stackrel{i B}{D}\right)} \varphi\right](x)=\frac{1}{\sqrt{B}} e^{\frac{D}{2 B} x^{2}}\left[\widehat{\mathrm{T}}_{\mathbf{L}} \varphi\right]\left(\frac{x}{B}\right),
$$

which involves the Laplace transform (3.8) of the function.

Of course, the interpretation (3.17) of (3.15) can as well be obtained by directly manipulating the expression of the transform. 


\subsection{Radial canonical transforms}

Besides the linear transforms, radial transforms involving (square-integrable) functions defined on the positive half-line $\mathbb{R}^{+}$can as well be associated with canonical transformations.

As theorized in [58], the radial $\mathrm{CT} \widehat{\mathrm{T}}_{\mathrm{M}}^{(n, m)}$, generated by the canonical transformation of the $n$ vectors $\widehat{\mathbf{x}}=\left\{\widehat{x}_{j}\right\}$ and $\widehat{\mathbf{p}}=\left\{\widehat{p}_{j}\right\}, j=1, \ldots, n$, which, preserving the Dirac brackets $\left[\widehat{x}_{j}, \widehat{p}_{k}\right]=i \delta_{j, k}$, is identified by a $2 n \times 2 n$ unimodular complex matrix $\mathbf{M}=\left(\begin{array}{l}\mathbf{A} \\ \mathbf{C}\end{array} \mathbf{D}\right)$, whose entries are (complex) multiplies of the $n \times n$ unit matrix such that $A D-B C=1$, amounts to the transform pair

$$
\begin{aligned}
& \widetilde{\varphi}(r)=\left[\widehat{\mathrm{T}}_{\mathrm{M}}^{(n, m)} \varphi\right](r)=\int_{0}^{+\infty} d r^{\prime} \mathrm{K}_{\mathrm{M}}^{(n, m)}\left(r, r^{\prime}\right) r^{\prime n-1} \varphi\left(r^{\prime}\right), \\
& \varphi\left(r^{\prime}\right)=\int_{\mathbb{C}^{+}} d \mu_{\mathrm{M}}^{(n, m)}(r) \mathrm{K}_{\mathrm{M}}^{(n, m)}\left(r, r^{\prime}\right)^{*} \widetilde{\varphi}(r),
\end{aligned}
$$

the kernel being

$$
\mathrm{K}_{\mathrm{M}}^{(n, m)}\left(r, r^{\prime}\right)=\frac{(-i)^{m+n / 2}}{B}\left(r r^{\prime}\right)^{1-n / 2} e^{\frac{i}{2 B}\left(A r^{2}+D r^{2}\right)} J_{n / 2+m-1}\left(\frac{r r^{\prime}}{B}\right) .
$$

By (3.19), functions $\varphi\left(r^{\prime}\right)$ in the Hilbert space $\mathcal{L}^{2}\left(\mathbb{R}^{+}\right)$with scalar product

$$
(\varphi, \psi)_{\mathcal{L}^{2}\left(\mathbb{R}^{+}\right)}=\int_{0}^{+\infty} r^{\prime n-1} \varphi^{*}\left(r^{\prime}\right) \psi\left(r^{\prime}\right) d r^{\prime},
$$

are transformed into functions $\widetilde{\varphi}(r)$, belonging to the space $\mathfrak{F}_{\mathrm{M}}^{(n, m)}$ of analytic functions of the complex variable $r$ restricted to the region $\mathbb{C}^{+}$, for which $\frac{\partial}{\partial r^{*}} \widetilde{\varphi}(r)=0$. Moreover, the space $\mathfrak{F}_{\mathbf{M}}^{(n, m)}$ is equipped with the scalar product

$$
(\widetilde{\varphi}, \widetilde{\psi})_{\widetilde{F}_{\mathbf{M}}^{(n, m)}}=\int_{\mathbb{C}^{+}} d \mu_{\mathbf{M}}^{(n, m)}(r) \widetilde{\varphi}^{*}(r) \widetilde{\psi}(r),
$$

the measure being

$$
d \mu_{\mathrm{M}}^{(n, m)}(r)=\frac{2}{\pi v} e^{\frac{1}{2 v}\left(u r^{2}+u^{*} r^{* 2}\right)}\left(r r^{*}\right)^{n / 2} K_{n / 2+m-1}\left(\frac{r r^{*}}{v}\right) d \Re r d \Im r,
$$

where $K_{\nu}$ denotes the MacDonald function of order $\nu$ [29], and, as before, $u=A^{*} D-B^{*} C \in \mathbb{C}$ and $v=2 \Im\left(B^{*} A\right) \in \mathbb{R}[57,58]$. It ensures that the transformed operators have the appropriate hermiticity properties and reproduce in the new variable the Schrödinger representation of the operators $\widehat{\mathbf{x}}^{2}=r^{2}, \widehat{\mathbf{x}} \cdot \widehat{\mathbf{p}}=-i r \frac{\partial}{\partial r}$ and $\widehat{\mathbf{p}}^{2}=-\left(\frac{\partial^{2}}{\partial r^{2}}+\frac{n-1}{r} \frac{\partial}{\partial r}+\frac{\lambda}{r^{2}}\right)$, with the real parameter $\lambda=-m(m+n-2), m=0,1,2, \ldots$, addressing the eigenvalue of the angular momentum $\widehat{L}^{2}=$ $\frac{1}{2} \sum \widehat{L}_{j k} \widehat{L}_{j k}, \widehat{L}_{j k}=\widehat{x}_{j} \widehat{p}_{k}-\widehat{x}_{k} \widehat{p}_{j}$, which is invariant under the unimodular transformation $\mathbf{M}$ [58].

In particular, with $n=2$ and $\mathbf{M}$ resorting to the entries of the Fourier transform matrix $\mathbf{F}$, one basically obtains the Hankel transform (2.34), whereas the fractional Fourier transform matrix $\mathbf{F}^{\alpha}$ yields through (3.20) the fractional Hankel transform (2.37).

The Barut-Girardello transform $\widehat{\mathbb{G}}_{n, m}$ represents an interesting example of complex radial CT. Resorting to the Bargmann matrix $\mathbf{B}$, it signifies [73]

$$
\begin{aligned}
{\left[\widehat{\mathbb{G}}_{n, m} \varphi\right](r) } & =\sqrt{2} \int_{0}^{+\infty} d r^{\prime}\left(r r^{\prime}\right)^{1-n / 2} e^{-\frac{1}{2}\left(r^{2}+r^{\prime 2}\right)} I_{n / 2+m-1}\left(\sqrt{2} r r^{\prime}\right) \varphi\left(r^{\prime}\right) \\
& =i^{n / 2+m-1}\left[\widehat{\mathbf{T}}_{\mathbf{B}}^{(n, m)} \varphi\right](r),
\end{aligned}
$$

$I_{\nu}$ denoting the modified Bessel function of the first kind of order $\nu$ : $J_{\nu}(i x)=i^{\nu} I_{\nu}(x)$ [29]. It was introduced in developing the formalism for coherent states associated with Lie algebras of non-compact groups, like, in particular, the semisimple Lie algebra $s o(2,1) \simeq \operatorname{sl}(2, \mathbb{R})$. As a direct generalization of the notion of coherent states associated with the Heisenberg algebra, such generalized coherent states were introduced as eigenstates of the lowering operator of the aforesaid algebra in the relative discrete representations $D^{ \pm}(j), j=-1 / 2,-1,-3 / 2, \ldots$ [73]. 


\subsection{Essentials of Hankel-type transforms}

The first and second Hankel-type transforms of Bessel order $\nu \geq-1 / 2$, depending on an arbitrary real parameter $\nu^{\prime}$, are respectively defined by $[74,75]$

$$
\begin{aligned}
& {\left[\widehat{\mathcal{H}}_{1, \nu, \nu^{\prime}} f\right](y) \equiv \widetilde{f}_{1, \nu, \nu^{\prime}}(y):=y^{1+2 \nu^{\prime}} \int_{0}^{+\infty}(x y)^{-\nu^{\prime}} J_{\nu}(x y) f(x) d x,} \\
& {\left[\widehat{\mathcal{H}}_{2, \nu, \nu^{\prime}} f\right](y) \equiv \widetilde{f}_{2, \nu, \nu^{\prime}}(y):=\int_{0}^{+\infty} x^{1+2 \nu^{\prime}}(x y)^{-\nu^{\prime}} J_{\nu}(x y) f(x) d x,}
\end{aligned}
$$

the function $f(x)$ being assumed to belong to the space $\mathcal{L}^{2}\left(\mathbb{R}^{+}\right)$.

It can be seen that they relate to each other according to

$$
\widehat{\mathcal{H}}_{1, \nu, \nu^{\prime}}^{\dagger}=\widehat{\mathcal{H}}_{2, \nu, \nu^{\prime}}, \quad \widehat{\mathcal{H}}_{2, \nu, \nu^{\prime}}^{\dagger}=\widehat{\mathcal{H}}_{1, \nu, \nu^{\prime}} .
$$

Also, as a consequence of the well-known orthogonality relation of the Bessel functions, both transforms are self-reciprocal:

$$
\widehat{\mathcal{H}}_{1, \nu, \nu^{\prime}}^{-1}=\widehat{\mathcal{H}}_{1, \nu, \nu^{\prime}}, \quad \widehat{\mathcal{H}}_{2, \nu, \nu^{\prime}}^{-1}=\widehat{\mathcal{H}}_{2, \nu, \nu^{\prime}},
$$

and separately obey the Parseval relations [76]

$$
\begin{aligned}
& \int_{0}^{+\infty} x^{-1-2 \nu^{\prime}} f^{*}(x) g(x) d x=\int_{0}^{+\infty} x^{-1-2 \nu^{\prime}} \widetilde{f}_{1, \nu, \nu^{\prime}}^{*}(x) \widetilde{g}_{1, \nu, \nu^{\prime}}(x) d x \\
& \int_{0}^{+\infty} x^{1+2 \nu^{\prime}} f^{*}(x) g(x) d x=\int_{0}^{+\infty} x^{1+2 \mu} \widetilde{f}_{2, \nu, \nu^{\prime}}^{*}(x) \widetilde{g}_{2, \nu, \nu^{\prime}}(x) d x
\end{aligned}
$$

along with the mixed relation

$$
\int_{0}^{+\infty} f^{*}(x) g(x) d x=\int_{0}^{+\infty} \widetilde{f}_{1, \nu, \nu^{\prime}}^{*}(x) \widetilde{g}_{2, \nu, \nu^{\prime}}(x) d x .
$$

In [77] the corresponding fractional order transforms have been introduced.

Notably, one can prove the identities [74, 75]

$$
\left[\widehat{\mathcal{H}}_{1, \nu, \nu^{\prime}} \widehat{B}_{\nu, \nu^{\prime}}^{\dagger} f\right](y)=-y^{2}\left[\widehat{\mathcal{H}}_{1, \nu, \nu^{\prime}} f\right](y), \quad\left[\widehat{\mathcal{H}}_{2, \nu, \nu^{\prime}} \widehat{B}_{\nu, \nu^{\prime}} f\right](y)=-y^{2}\left[\widehat{\mathcal{H}}_{2, \nu, \nu^{\prime}} f\right](y),
$$

involving the Bessel-type differential operator $\widehat{B}_{\nu, \nu^{\prime}}$ and its adjoint $\widehat{B}_{\nu, \nu^{\prime}}^{\dagger}$ :

$$
\begin{aligned}
\widehat{B}_{\nu, \nu^{\prime}} & :=\frac{d^{2}}{d x^{2}}+\left(1+2 \nu^{\prime}\right) \frac{1}{x} \frac{d}{d x}+\left(\nu^{\prime 2}-\nu^{2}\right) \frac{1}{x^{2}}=x^{-\nu^{\prime}-\nu-1} \frac{\partial}{\partial x} x^{2 \nu+1} \frac{\partial}{\partial x} x^{\nu^{\prime}-\nu}, \\
\widehat{B}_{\nu, \nu^{\prime}}^{\dagger} & :=\frac{d^{2}}{d x^{2}}-\left(1+2 \nu^{\prime}\right) \frac{1}{x} \frac{d}{d x}+\left[\left(\nu^{\prime}+1\right)^{2}-\nu^{2}\right] \frac{1}{x^{2}}=x^{\nu^{\prime}-\nu} \frac{\partial}{\partial x} x^{2 \nu+1} \frac{\partial}{\partial x} x^{-\nu^{\prime}-\nu-1} .
\end{aligned}
$$

Relations (3.23) can be used, for instance, to write the solution of the evolutionary equation $[74,75]$

$$
\kappa \frac{\partial}{\partial \tau} h(x, \tau)=\widehat{B}_{\nu, \nu^{\prime}}^{\dagger} h(x, \tau),
$$

satisfying the initial condition $h(x, 0)=f(x)$, in the transform conjugate $y$-space as $\widetilde{h}_{1, \nu, \nu^{\prime}}(y, \tau)=$ $e^{-(\tau / \kappa) y^{2}} \widetilde{f}_{1, \nu, \nu^{\prime}}(y)$ for arbitrary values of $\kappa$. Then, transforming back to the $x$-space, we obtain

$$
h(x, \tau)=\frac{\kappa}{2 \tau} x^{1+2 \nu^{\prime}} \int_{0}^{+\infty}(x y)^{-\nu^{\prime}} e^{-\frac{\kappa}{4 \tau}\left(x^{2}+y^{2}\right)} I_{\nu}\left(\frac{\kappa}{2 \tau} x y\right) f(y) d y
$$

under the condition that $|\arg (\tau / \kappa)|<\pi / 4$, which for both $\tau$ and $\kappa$ real turns into $\tau / \kappa>0$. 
As noted in [77], on account of the symbolic solution $h(x, \tau)=\exp \left[(\tau / \kappa) \widehat{B}_{\nu, \nu^{\prime}}^{\dagger}\right] f(x)$ of $(3.24)$, the above yields an explicit functional representation of the exponential operator $\exp \left[\beta \widehat{B}_{\nu, \nu^{\prime}}^{\dagger}\right]$ :

$$
\left[e_{\nu, \nu^{\prime}}^{\beta \widehat{B}^{\dagger}} f\right](x)=\frac{1}{2 \beta} x^{1+2 \nu^{\prime}} \int_{0}^{+\infty}(x y)^{-\nu^{\prime}} e^{-\frac{1}{4 \beta}\left(x^{2}+y^{2}\right)} I_{\nu}\left(\frac{x y}{2 \beta}\right) f(y) d y
$$

For $\beta=i / 2$ it eventually conveys a representation of the transform $\widehat{\mathcal{H}}_{1, \nu, \nu^{\prime}}$ as a symmetric product of exponentials of the $s u(1,1)$ algebra generators, similar to $(2.35)$ for the Hankel transforms:

$$
\widehat{\mathcal{H}}_{1, \nu, \nu^{\prime}}=i^{\nu+1} e^{-\frac{i}{2} x^{2}} e^{\frac{i}{2} \widehat{B}_{\nu, \nu^{\prime}}^{\dagger}} e^{-\frac{i}{2} x^{2}}=i^{\nu+1} e^{-i \frac{\pi}{2}\left[\widehat{\mathrm{K}}_{-}^{(1)}+\widehat{\mathrm{K}}_{+}^{(1)}\right]} .
$$

This is not surprising since the operators

$$
\widehat{\mathrm{K}}_{+}^{(1)}:=\frac{1}{2} x^{2}, \quad \widehat{\mathrm{K}}_{-}^{(1)}:=-\frac{1}{2} \widehat{B}_{\nu, \nu^{\prime}}^{\dagger}, \quad \widehat{\mathrm{K}}_{3}^{(1)}:=-\frac{i}{2}\left(x \frac{d}{d x}-\nu^{\prime}\right),
$$

constitute a non self-adjoint one-variable realization of the $s u(1,1)$ algebra generators obeying, as it should be, the inherent commutation relations shown in (2.4).

The adjoint operators

$$
\widehat{\mathrm{K}}_{+}^{(2)}:=\frac{1}{2} x^{2}, \quad \widehat{\mathrm{K}}_{-}^{(2)}:=-\frac{1}{2} \widehat{B}_{\nu, \nu^{\prime}}, \quad \widehat{\mathrm{K}}_{3}^{(2)}:=-\frac{i}{2}\left(x \frac{d}{d x}+\nu^{\prime}+1\right)
$$

pertain to the second Hankel-type transform $\widehat{\mathcal{H}}_{2, \nu, \nu^{\prime}}$, which is in fact amenable for a factored form representation analogous to (3.26) in terms of them [77].

With $\nu^{\prime}=-1$ and $\nu=m$ the operators (3.27) turn into (2.29), pertaining to the Hankel transform $\widehat{\mathcal{H}}_{m}$ of order $m$, considered in Section 2.2 in connection with the propagation problem in circular cylindrical coordinates; indeed, $\widehat{\mathcal{H}}_{1, \nu, \nu^{\prime}} \rightarrow \widehat{\mathcal{H}}_{m}$ for the identified values of $\nu$ and $\nu^{\prime}$.

In general, as noted in [77], for suitable values of $\nu^{\prime}$ and $\nu$ the transforms $\widehat{\mathcal{H}}_{1, \nu, \nu^{\prime}}$ and $\widehat{\mathcal{H}}_{2, \nu, \nu^{\prime}}$ can be framed within the formalism of the radial CTs, as theorized in [58] and shortly reviewed in Section 3.2. In fact, if the $2 n \times 2 n$ transformation matrix resorts to the entries of the Fourier matrix $\mathbf{F}$, the kernel (3.20) becomes

$$
\mathrm{K}_{\mathbf{F}}^{(n, m)}\left(r, r^{\prime}\right)=(-i)^{m+n / 2}\left(r r^{\prime}\right)^{1-n / 2} J_{n / 2+m-1}\left(r r^{\prime}\right),
$$

the resulting transform being seen as the radial part of a $n$-dimensional linear CT, specifically representing a $\pi / 2$-rotation for each pair of the canonically conjugate operators in the relevant $n$-component position and momentum operator vectors. The kernel $\mathrm{K}_{\mathrm{F}}^{(n, m)}$ resembles that of the transforms (3.22) for suitable settings of the parameters $\nu$ and $\nu^{\prime}$ in terms of the inherent dimension $n$ and eigenvalue $\lambda$. In fact, one respectively has $n=-2 \nu^{\prime}$ and $n=2\left(1+\nu^{\prime}\right)$, which so are meaningful for $\nu^{\prime}$ integer or half-integer (negative or positive), whereas correspondingly $m=\nu+\nu^{\prime}+1$ and $m=\nu-\nu^{\prime}$, thus yielding $\lambda=\left(\nu^{\prime}+1\right)^{2}-\nu^{2}$ and $\lambda=\nu^{\prime 2}-\nu^{2}$.

Disregarding the specific link of the canonical transforms to the canonical transformations as briefly reviewed above, we rewrite the integral transforms (3.22) through the formal relations

$$
\begin{aligned}
& {\left[\widehat{\mathcal{H}}_{1, \nu, \nu^{\prime}} \varphi\right](r)=i^{1+\nu} \int_{0}^{\infty} \mathrm{K}_{\mathbf{F}}^{\left(-2 \nu^{\prime}, \nu+\nu^{\prime}+1\right)}\left(r, r^{\prime}\right) r^{\prime-1-2 \nu^{\prime}} \varphi\left(r^{\prime}\right) d r^{\prime},} \\
& {\left[\widehat{\mathcal{H}}_{2, \nu, \nu^{\prime}} \varphi\right](r)=i^{1+\nu} \int_{0}^{\infty} \mathrm{K}_{\mathbf{F}}^{\left(2\left(1+\nu^{\prime}\right), \nu-\nu^{\prime}\right)}\left(r, r^{\prime}\right) r^{\prime 1+2 \nu^{\prime}} \varphi\left(r^{\prime}\right) d r^{\prime},}
\end{aligned}
$$

which, contrary to the general account (3.19), should be intended as transforming functions of a real variable into functions of a real variable. 
In virtue of the analysis presented in Section 2, one may guess that the above might be of relevance in connection with the Appell transformation for a "radial PWE" in a higher-dimension domain.

In Section 4.3 we will prove that the "Laplace-like" versions of (3.29) relate to the Appell transformation for the radial $\mathrm{HE}$, which is seen to connect temperature functions evolving from functions linked through the aforesaid transforms.

\section{Caloric Appell transformation: Laplace and Hankel-type transforms}

In virtue of the well-known analogy between the 1D HE and the 2D linear PWE, we will properly reproduce within the context of the $1 \mathrm{D} \mathrm{HE}$ the interpretation of the Appell transformation, as elaborated in Section 2.1.2 in connection with the optical propagation. We will find that the well-known caloric Appell transformation connects temperature functions resulting from initial conditions linked by a Laplace-like similarity transformation. Thus, as the optical Appell transformation is understood to manifest the action of the evolving-location Fourier transform operator on wavefunctions, likewise the caloric Appell transformation will be seen to result from the action of the evolving-time Laplace-like transform operator on temperature functions. A similar relation will then be shown to hold also for the Appell transformation for the radial HE, accordingly resorting to suitably defined Hankel-type transforms, explicable as "radialLaplace"-type transforms.

\subsection{D heat equation}

The $1 \mathrm{D} \mathrm{HE}{ }^{9}$

$$
2 \frac{\partial}{\partial t} u(x, t)=\frac{\partial^{2}}{\partial x^{2}} u(x, t)
$$

has been the object of extensive studies; few titles from the wide bibliography are in $[1,2,3$, $4,5,6,7]$. See also $[12,13,14]$. The temperature or caloric function $u(x, t)$ is usually assumed to be of class $C^{1}$ with respect to $t$ and of class $C^{2}$ with respect to $x$ across the region $\mathcal{D}$ in the $x, t$-plane where (4.1) holds [2]. In view of the analysis we will perform, $u(x, t)$ will be here supposed to be of class $C^{\infty}$ with respect to the variables $x, t$, which will be considered as real unitless variables.

As is well known, solutions to (4.1) can be obtained as the Poisson transform of the initial conditions, that is $[2]$

$$
f(x, t)=\left[\widehat{\mathcal{U}}_{\mathrm{HE}}(t) f_{0}\right](x):=\frac{1}{\sqrt{2 \pi t}} \int_{-\infty}^{\infty} e^{-\frac{(x-y)^{2}}{2 t}} f_{0}(y) d y
$$

with $f_{0}(x)=f(x, 0)$, under the general statement that $f_{0}(x)$ be Lebesgue integrable in every finite interval. The integral kernel reproduces the fundamental solution of (4.1):

$$
S(x, t):=\frac{1}{\sqrt{2 \pi t}} e^{-\frac{x^{2}}{2 t}}, \quad t>0 .
$$

\footnotetext{
${ }^{9}$ Actually, the standard form of the $1 \mathrm{D} \mathrm{HE}$ is [2]

$$
\frac{\partial}{\partial t} u(x, t)=\frac{\partial^{2}}{\partial x^{2}} u(x, t)
$$
}

Here, in order to favor the comparison with the 2D PWE, we will work with (4.1), which amounts to the trivial variable scaling: $t \rightarrow t / 2$ between the "time" in the standard form and that in (4.1). 
Resorting to the review in Section 3.1, we see that $\widehat{\mathcal{U}}_{\mathrm{HE}}=\widehat{\mathrm{T}}_{\mathrm{P}}$, with the replacement $\tau \rightarrow t$ in the relevant canonical transformation matrix P. Evidently, the Poisson transform (4.2) corresponds to the Fresnel transform (2.3) by the variable interchange $t \leftrightarrow i \zeta$, which is the basis of the analogy between the 1D HE and the 2D PWE. It conveys an integral transform representation for the "evolution" operator $\widehat{\mathcal{U}}_{\mathrm{HE}}(t)$ associated with (4.1), which can as well be given the exponential operator representation

$$
\widehat{\mathcal{U}}_{\mathrm{HE}}(t):=e^{\frac{t}{2} \frac{d^{2}}{d x^{2}}}=e^{-t \widehat{\mathrm{K}}_{-}},
$$

in analogy with that of the optical propagator $\widehat{\mathcal{U}}_{\text {PWE }}(\zeta)$. As in that case, we consider the two representations as fully equivalent, disregarding indeed the question of specifying the domain of functions over which such an equivalence effectively holds [2].

We have expressed $\widehat{\mathcal{U}}_{\mathrm{HE}}(t)$ in terms of the operator $\widehat{\mathrm{K}}_{-}$, thus signalizing that in connection with the 1D HE we will resort to the same basis operators (2.4) as for the 2D PWE, although linear combinations of them by in general complex coefficients will more properly be considered. This will amount to deal with complex canonical transforms. In fact, on discussing the caloric Appell transformation we will be led to consider the Laplace transform represented by the complex symplectic matrix L (Section 3.1.1), whereas the afore-discussed optical Appell transformation has led to the Fourier transform, which in turn resorts to the real symplectic matrix F (Section 2.1.2).

\subsection{Some about the heat polynomials and the associated functions}

The heat polynomials $v_{n}(x, t)$ are defined as the polynomial solutions of (4.1), which realize the power series expansion of the simple exponential solution [2], i.e.

$$
e^{\chi x+\chi^{2} t / 2}=\sum_{n=0}^{\infty} \frac{\chi^{n}}{n !} v_{n}(x, t),
$$

with $\chi$ arbitrary parameter. They explicitly write as

$$
v_{n}(x, t):=n ! \sum_{j=0}^{[n / 2]} \frac{t^{j}}{2^{j} j !(n-2 j) !} x^{n-2 j},
$$

which reveals their connection with the Hermite polynomials as $v_{n}(x, t)=(-t / 2)^{n / 2} H_{n}(x / \sqrt{-2 t})$.

Interestingly, the $v_{n}$ s solve (4.1) subject to monomial initial distributions $v_{n}(x, 0)=x^{n}$, being [2]

$$
v_{n}(x, t)=\frac{1}{\sqrt{2 \pi t}} \int_{-\infty}^{\infty} e^{-\frac{(x-y)^{2}}{2 t}} y^{n} d y, \quad 0<t<\infty .
$$

In addition, they obey the second-order differential equations

$$
\left(t \frac{d^{2}}{d x^{2}}+x \frac{d}{d x}-n\right) v_{n}(x, t)=0
$$

which determine the $x$-dependence of the $v_{n} \mathrm{~s}$, whose complete expressions follow then from the inherent initial conditions $v_{n}(x, 0)=x^{n}$.

The associated functions $w_{n}(x, t)$, which are solutions of the HE as well, are obtained from the $v_{n} \mathrm{~s}$ by the caloric Appell transformation $[1,2]$ :

$$
w_{n}(x, t):=S(x, t) v_{n}\left(\frac{x}{t},-\frac{1}{t}\right)=t^{n} S(x, t) v_{n}(x,-t) .
$$


They admit the generating function relation

$$
\frac{1}{\sqrt{2 \pi t}} e^{-\frac{(x-\chi)^{2}}{2 t}}=\sum_{n=0}^{\infty} \frac{\chi^{n}}{n !} w_{n}(x, t)=S(x-\chi, t),
$$

which parallels (4.4), as well as, in analogy with (4.5), the integral representation [2]

$$
w_{n}(x, t)=\frac{1}{2 \pi} \int_{-\infty}^{\infty} e^{-\frac{t}{2} y^{2}+i x y}(-i y)^{n} d y
$$

Finally, the $w_{n}$ s obey the differential equation

$$
\left(t \frac{d^{2}}{d x^{2}}+x \frac{d}{d x}+n+1\right) w_{n}(x, t)=0 .
$$

We address the reader to the bibliography $[2,3,4,5]$ for a more complete account of the topic.

In [9] the optical analogs of the heat polynomials and the associated functions have been deduced, which have suggested the interpretation of the optical Appell transformation in terms of Fourier-related source functions, as illustrated in Section 2.1.2.

\subsection{Laplace transform and Appell transformation for the 1D heat equation}

As earlier remarked, the suitable Lie-algebra context should be now that of the six-parameter algebra $s l(2, \mathbb{C})$; accordingly, taking account also of the Weyl algebra, we should deal with the semigroup $W S L(2, \mathbb{C})$. However, in our analysis we will make use of CTs of the type (3.15) (associated with matrices of the form (3.14)). Hence, we can limit ourselves to deal with $S L(2, \mathbb{C})$, even better with the subgroup $\subset S L(2, \mathbb{C})$ formed by the matrices $(3.14)$, that amounts to a subalgebra $\subset \operatorname{sl}(2, \mathbb{C})$, spanned by the basis operators $\left\{\widehat{K}_{+}, \widehat{K}_{3}, \widehat{K}_{-}\right\}$through linear combinations with coefficients respectively of the type $\{i \alpha, \gamma, i \beta\}$ with $\alpha, \beta$, $\gamma$ real.

We consider for every operator $\widehat{\mathrm{K}}$ in the aforesaid subalgebra $\subset \operatorname{sl}(2, \mathbb{C})$ the relevant "dual" operator $\widehat{\widetilde{\mathrm{K}}}=\widehat{\mathrm{T}}_{\mathbf{L}} \widehat{\mathrm{K}}_{\mathbf{L}}^{-1}$, obtained from the former by a similarity transformation involving the Laplace-like operator $\widehat{T}_{\mathbf{L}}$. Due to $(3.11)$ or $(3.12)$, $\widehat{\widetilde{K}}$ belongs to the same subalgebra as $\widehat{K}$.

By retracing the eigenstate-based procedure [10, 12] applied to the 2D PWE in Section 2.1.2, we consider the eigenvalue problems for $\widehat{K}$, as represented by the equivalent eigenvalue equations

$$
\widehat{\mathrm{K}} f_{\lambda}(x)=\lambda f_{\lambda}(x)
$$

or

$$
\widehat{\mathrm{K}}(t) v_{\lambda}(x, t)=\lambda v_{\lambda}(x, t),
$$

and for $\widehat{\widetilde{K}}$, as similarly described by

$$
\widehat{\widetilde{\mathrm{K}}} g_{\lambda}(x)=\lambda g_{\lambda}(x) \text {, }
$$

or

$$
\widehat{\widetilde{\mathrm{K}}}(t) w_{\lambda}(x, t)=\lambda w_{\lambda}(x, t) .
$$

Here, the evolving-time operators explicitly mean

$$
\widehat{\mathrm{K}}(t):=\widehat{\mathcal{U}}_{\mathrm{HE}}(t) \widehat{\mathrm{K}} \widehat{\mathcal{U}}_{\mathrm{HE}}^{-1}(t)=e^{-t \widehat{\mathrm{K}}_{-}} \widehat{\mathrm{K}} e^{t \widehat{\mathrm{K}}_{-}} .
$$


Just as for the PWE, the above equations signify that we are looking for temperature functions $v(x, t)$ and $w(x, t)$ corresponding to initial conditions linked by the integral transform (3.10), i.e. $v(x, 0)=f(x)$ and $w(x, 0)=g(x)=\left[\widehat{\mathrm{T}}_{\mathbf{L}} f\right](x)$, with in particular $f(x)$ being identified as an eigenstate of a given operator in the subalgebra of concern. Hence, as $v_{\lambda}(x, t)=e^{-t \widehat{\mathrm{K}}_{-}} f_{\lambda}(x)$, the $w_{\lambda} \mathrm{s}$ are in turn obtained from the $v_{\lambda} \mathrm{s}$ by the "instantaneous" transformation

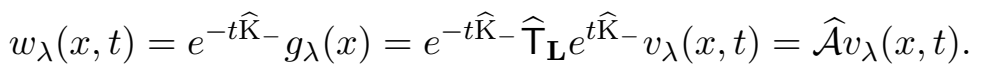

The inherent symmetry operator $\widehat{\mathcal{A}}$, identified by the same symbol as the optical Appell operator,

$$
\widehat{\mathcal{A}}:=e^{-t \widehat{\mathrm{K}}_{-}} \widehat{\mathrm{T}}_{\mathbf{L}} e^{t \widehat{\mathrm{K}}_{-}}=\widehat{\mathrm{T}}_{\mathbf{L}}(t),
$$

signifies now the evolving-time Laplace operator. Its explicit dependence on $t$ will be omitted.

Contrary to the optical operator, the caloric Appell operator individualizes the complex canonical transformation matrix

$$
\mathbf{M}_{\widehat{\mathcal{A}}}:=\left(\begin{array}{cc}
1 & -i t \\
0 & 1
\end{array}\right)\left(\begin{array}{ll}
0 & i \\
i & 0
\end{array}\right)\left(\begin{array}{cc}
1 & i t \\
0 & 1
\end{array}\right)=\left(\begin{array}{cc}
t & i\left(1+t^{2}\right) \\
i & -t
\end{array}\right)
$$

Being it of the type (3.14), we can apply the recipe (3.17) with $\tau_{0}=t$, thus inferring the associated functions $w_{\lambda} \mathrm{S}$ from the $v_{\lambda} \mathrm{S}$ in accord to the Appell rule (4.7) (apart from the factor $1 / \sqrt{2 \pi}$ ):

$$
w_{\lambda}(x, t):=\widehat{\mathcal{A}} v_{\lambda}(x, t)=\frac{1}{\sqrt{t}} e^{-\frac{x^{2}}{2 t}} v_{\lambda}\left(\frac{x}{t},-\frac{1}{t}\right) .
$$

By (3.18) one recovers the primary relation between the "source" functions, i.e. $g(x)=\left[\widehat{\mathbf{T}}_{\mathbf{L}} f\right](x)$.

Notably, disregarding the formal complexity of the inverse transform and naïvely using the inverse matrix $\mathbf{M}_{\widehat{\mathcal{A}}^{-1}}=\left(\begin{array}{c}-t \\ -i\end{array}{ }_{t}^{-i\left(1+t^{2}\right)}\right)$, one obtains the correct rule to recover the $v$ s from the $w \mathrm{~s}$.

As a basic example, we may consider the heat polynomials and the associated functions. As signalized by (4.5), the former are caloric functions arising from the diffusion of the monomials $x^{n}$ s. Therefore, the eigenvalue equations (4.9) and (4.10) must be specialized for the scale transform generator $\widehat{\mathrm{K}}_{3}$ :

$$
\widehat{\mathrm{K}}_{3} x^{n}=\lambda x^{n},
$$

with $\lambda=-i(n+1 / 2), n=0,1,2, \ldots$, and for the relevant evolving-time operator $\widehat{\mathrm{K}}_{3}(t)$ :

$$
\widehat{\mathrm{K}}_{3}(t) v_{n}(x, t)=\left[\widehat{\mathrm{K}}_{3}+i t \widehat{\mathrm{K}}_{-}\right] v_{n}(x, t)=\lambda v_{n}(x, t),
$$

eventually resulting into (4.6), obeyed by the $v_{n}$ s.

As to the dual operator $\widehat{\widetilde{K}}_{3}=\widehat{\mathrm{T}}_{\mathbf{L}} \widehat{\mathrm{K}}_{3} \widehat{\mathrm{T}}_{\mathbf{L}}^{-1}=-\widehat{\mathrm{K}}_{3}$, the eigenvalue equation (4.11) writes as

$$
\widehat{\mathrm{K}}_{3} g_{\lambda}=-\lambda g_{\lambda}
$$

whereas (4.12) for the evolving-time operator $\widehat{\widetilde{\mathrm{K}}}_{3}(t)=-\widehat{\mathrm{K}}_{3}-i t \widehat{\mathrm{K}}_{-}$specializes as

$$
\left[\widehat{\mathrm{K}}_{3}+i t \widehat{\mathrm{K}}_{-}\right] w_{n}(x, t)=\frac{i}{2}\left(n+\frac{1}{2}\right) w_{n}(x, t),
$$

which, as expected, yields (4.8) obeyed by the associated functions $w_{n}$.

Thus, the caloric Appell transformation of the heat polynomials (which, as seen, are associated with the eigenvalue problem for $\widehat{\mathrm{K}}_{3}$ with $\left.\lambda=-i(n+1 / 2), n=0,1,2, \ldots\right)$ yields the 
temperature functions associated with the eigenvalue problem for the Laplace dual operator $\widehat{\widetilde{K}}_{3}=\widehat{\mathrm{T}}_{\mathbf{L}} \widehat{\mathrm{K}}_{3} \widehat{\mathrm{T}}_{\mathbf{L}}^{-1}$, or equivalently with the eigenvalue problem for $\widehat{\mathrm{K}}_{3}$ with opposite eigenvalues.

Notably, since the Fourier and Laplace dual operators of $\widehat{\mathrm{K}}_{3}$ are the same: $\widehat{\mathcal{F}} \widehat{\mathrm{K}}_{3} \widehat{\mathcal{F}}^{-1}=$ $\widehat{\mathrm{T}}_{\mathbf{L}} \widehat{\mathrm{K}}_{3} \widehat{\mathrm{T}}_{\mathbf{L}}^{-1}=-\widehat{\mathrm{K}}_{3}$, as discussed in [9], the Appell transformation of the heat polynomials can as well be associated with a Fourier-similarity relation, whilst in general, as seen above, one must resort to a Laplace-similarity relation when dealing with generic temperature functions.

In order to complete the correspondence with the 2D PWE, we can say that a fractional caloric Appell transformation may also be introduced as the evolving-time fractional Laplace transform operator

$$
\widehat{\mathcal{A}}^{\alpha}:=e^{-t \widehat{\mathrm{K}}_{-}} \widehat{\mathrm{T}}_{\mathbf{L}^{\alpha}} e^{t \widehat{\mathrm{K}}_{-}}=\widehat{\mathrm{T}}_{\mathbf{L}^{\alpha}}(t),
$$

with $\widehat{T}_{\mathbf{L}^{\alpha}}$ given as discussed in Section 3.1.1. Evidently, it identifies the transformation matrix

$$
\mathbf{M}_{\widehat{\mathcal{A}}^{\alpha}}=\left(\begin{array}{cc}
1 & -i t \\
0 & 1
\end{array}\right)\left(\begin{array}{cc}
\cos \phi & i \sin \phi \\
i \sin \phi & \cos \phi
\end{array}\right)\left(\begin{array}{cc}
1 & i t \\
0 & 1
\end{array}\right)=\left(\begin{array}{cc}
\cos \phi+t \sin \phi & i\left(1+t^{2}\right) \sin \phi \\
i \sin \phi & \cos \phi-t \sin \phi
\end{array}\right)
$$

and hence maps temperature functions into temperatures functions according to

$$
w(x, t)=\widehat{\mathcal{A}}^{\alpha} v(x, t)=\frac{1}{\sqrt{\cos \phi+t \sin \phi}} e^{-\frac{\sin \phi}{2(\cos \phi+t \sin \phi)} x^{2}} v\left(\frac{x}{\cos \phi+t \sin \phi}, \frac{t \cos \phi-\sin \phi}{\cos \phi+t \sin \phi}\right) .
$$

\subsection{Hankel-type transform and Appell transformation for the radial heat equation}

The radial HE is signified by

$$
2 \frac{\partial}{\partial t} u(r, t)=\widehat{D}_{\mu} u(r, t)
$$

where $\mu$ is an arbitrary real parameter and $\widehat{D}_{\mu}$ denotes the differential operator $\widehat{D}_{\mu}:=\frac{\partial^{2}}{\partial r^{2}}+$ $\frac{\mu-1}{r} \frac{\partial}{\partial r}$ [78]. When $\mu=n$, a positive integer, $\widehat{D}_{n}$ becomes the Laplacian operator in the radial coordinate appropriate to a $n$-dimensional Euclidean space: $\widehat{D}_{n}=\frac{\partial^{2}}{\partial x_{1}^{2}}+\cdots+\frac{\partial^{2}}{\partial x_{n}^{2}}$. As before, we consider both $r \in[0,+\infty)$ and $t$ as real unitless variables; also, as in (4.1), for computational convenience we have added the factor 2 with respect to the equation appearing in the literature, thus implying the "time" scaling: $t \rightarrow t / 2$ with respect to that equation.

For $\mu>1,(4.13)$ admits as solutions the radial heat polynomials $R_{n, \mu}(r, t), n=0,1,2 \ldots$, and their Appell transforms $\widetilde{R}_{n, \mu}(r, t)$, explicitly given by [78]

$$
\begin{aligned}
& R_{n, \mu}(r, t):=2^{n} n ! t^{n} L_{n}^{\mu / 2-1}\left(-\frac{r^{2}}{2 t}\right), \\
& \widetilde{R}_{n, \mu}(r, t):=S_{\mu}(r, t) R_{n, \mu}\left(\frac{r}{t},-\frac{1}{t}\right)=t^{-2 n} S_{\mu}(r, t) R_{n, \mu}(r,-t),
\end{aligned}
$$

where $S_{\mu}(r, t)$ denotes the fundamental solution of (4.13):

$$
S_{\mu}(r, t):=\frac{1}{(2 \pi t)^{\mu / 2}} e^{-\frac{r^{2}}{2 t}}
$$

With $\mu=2$ we can recognize a formal correspondence between (4.13) and the 2D PWE (2.28) with $m=0$, which so rules the evolution of the radial wavefunction for a circularly symmetric wavefield. Indeed, just as solutions to the 2D radial PWE follow from the Hankel-Poisson 
integral (2.30) under definite initial conditions, likewise solutions to (4.13) may be obtained from assigned initial conditions $u(r, 0)=\varphi(r)$ by the integral transform

$$
u(r, t)=\int_{0}^{+\infty} \mathrm{K}_{\mu}\left(r, r^{\prime} ; t\right) \varphi\left(r^{\prime}\right) d r^{\prime}
$$

with

$$
\mathrm{K}_{\mu}\left(r, r^{\prime} ; t\right):=\frac{1}{t} r^{1-\mu / 2} r^{\prime \mu / 2} e^{-\frac{1}{2 t}\left(r^{2}+r^{\prime 2}\right)} I_{\mu / 2-1}\left(\frac{r r^{\prime}}{t}\right),
$$

which yields, for instance, the heat polynomials $R_{n, \mu}(r, t)$ with $\varphi(r)=r^{2 n}$.

More precisely, we may recognize a correspondence with the Hankel-type transforms, discussed in Section 3.3, which, as there noted, for specific values of the relevant parameters yield the conventional Hankel transform, or more general (real and complex) radial transforms. In fact, on account of the above clarified link between the 1D HE and the Laplace transform, it is natural to consider the kernel one would obtain from (3.20) with the entries of the Laplace matrix $\mathbf{L}$,

$$
\mathrm{K}_{\mathbf{L}}^{(n, m)}\left(r, r^{\prime}\right)=(-)^{m+n / 2}\left(r r^{\prime}\right)^{1-n / 2} I_{n / 2+m-1}\left(r r^{\prime}\right),
$$

which then, through (3.19), should yield a sort of "radial" Laplace transform. More in general, using it in (3.19) with the aforestated correspondences $(n, m) \leftrightarrow\left(\nu, \nu^{\prime}\right)$, one would obtain in a sense the complex versions of the transforms $\widehat{\mathcal{H}}_{1, \nu, \nu^{\prime}}$ and $\widehat{\mathcal{H}}_{2, \nu, \nu^{\prime}}$, i.e.

$$
\begin{aligned}
& {\left[\widehat{\mathcal{L}}_{1, \nu, \nu^{\prime}} \varphi\right](r):=(-)^{1+\nu} r^{1+2 \nu^{\prime}} \int_{0}^{+\infty}\left(r r^{\prime}\right)^{-\nu^{\prime}} I_{\nu}\left(r r^{\prime}\right) \varphi\left(r^{\prime}\right) d r^{\prime}} \\
& {\left[\widehat{\mathcal{L}}_{2, \nu, \nu^{\prime}} \varphi\right](r):=(-)^{1+\nu} \int_{0}^{+\infty} r^{1+2 \nu^{\prime}}\left(r r^{\prime}\right)^{-\nu^{\prime}} I_{\nu}\left(r r^{\prime}\right) \varphi\left(r^{\prime}\right) d r^{\prime}}
\end{aligned}
$$

Clearly, they relate to the Laplace transform just as the Barut-Girardello transform (3.21) relates to the Bargmann transform (3.5). In this connection, we mention that in [77], reproducing the structure of the transforms (3.22), two analogous Barut-Girardello-type transforms have been introduced along with the relevant fractional-order versions.

It is easy to see that relation $(3.25)$ yields for $\widehat{\mathcal{L}}_{1, \nu, \nu^{\prime}}$ the operator representation

$$
\widehat{\mathcal{L}}_{1, \nu, \nu^{\prime}}=e^{-\widehat{\mathrm{K}}_{+}^{(1)}} e^{\widehat{\mathrm{K}}_{-}^{(1)}} e^{-\widehat{\mathrm{K}}_{+}^{(1)}}=e^{(\pi / 2)\left[\widehat{\mathrm{K}}_{-}^{(1)}-\widehat{\mathrm{K}}_{+}^{(1)}\right]}
$$

in terms of the operators (3.27); of course, a similar representation can be obtained for the adjoint transform $\widehat{\mathcal{L}}_{2, \nu, \nu^{\prime}}$ in terms of the operators (3.28).

The correspondence between (4.17) and (3.11) is evident.

It is also evident that relation (3.25) (as that involving the adjoint operator $\widehat{B}_{\nu, \nu^{\prime}}$ ) can be made to reproduce the general expression (4.15) for the solutions of the radial HE by setting $\nu=\mu / 2-1$ and $\nu^{\prime}=-\mu / 2$ (as correspondingly, $\nu=\nu^{\prime}=\mu / 2-1$ ). In fact, writing, for instance:

$$
\widehat{\mathcal{U}}_{\mathrm{RHE}}(t):=e^{-t \widehat{\mathrm{K}}_{-}^{(1)}},
$$

with $\widehat{\mathrm{K}}_{-}^{(1)}=-\frac{1}{2} \widehat{B}_{\mu / 2-1,-\mu / 2}^{\dagger}=-\frac{1}{2} \widehat{D}_{\mu}$, we see that, by

$$
u(r, t)=\left[\widehat{\mathcal{U}}_{\mathrm{RHE}}(t) \varphi\right](r),
$$

one recovers (4.15) on account of (3.25). 
Then, just reproducing the considerations previously developed in connection with the $2 \mathrm{D}$ PWE and the 1D HE, in the light of the correspondence of (4.17) to (3.11) and of (4.18) to (4.3), we can argue that the Appell transformation of solutions of the radial HE arises from the action of the evolving-time "radial-Laplace"-type transform operator $\widehat{\mathcal{L}}_{1, \mu / 2-1,-\mu / 2}(t)$ on temperature functions. Explicitly, the relevant Appell operator $\widehat{\mathcal{A}}_{\mu}$ is to be intended as

$$
\widehat{\mathcal{A}}_{\mu}:=e^{-t \widehat{\mathrm{K}}_{-}^{(1)}} \widehat{\mathcal{L}}_{1, \mu / 2-1,-\mu / 2} e^{t \widehat{\mathrm{K}}_{-}^{(1)}}=\widehat{\mathcal{L}}_{1, \mu / 2-1,-\mu / 2}(t),
$$

which amounts to the parallel "heat conduction" problems: $u(r, t)=e^{-t \widehat{\mathrm{K}}_{-}^{(1)}} \varphi(r)$ and $w(r, t)=$ $e^{-t \widehat{\mathrm{K}}_{-}^{(1)}} \psi(r)$, with $\psi(r)=\widehat{\mathcal{L}}_{1, \mu / 2-1,-\mu / 2} \varphi(r)$.

The direct evaluation or the use of the matrix-based procedure leads to the well-known recipe (see $(4.14))$ :

$$
\widehat{\mathcal{A}}_{\mu} u(r, t)=t^{-\mu / 2} e^{-\frac{r^{2}}{2 t}} u\left(\frac{r}{t},-\frac{1}{t}\right) \propto S_{\mu}(r, t) u\left(\frac{r}{t},-\frac{1}{t}\right),
$$

thus confirming the interpretation (4.19).

Needless to say, as before a fractional Appell transformation can as well be introduced, by exploiting the fractional versions of the transforms (4.16), inspired by those of the Hankel-type transforms [77].

\section{Concluding notes}

After reviewing the interpretation of the optical Appell transformation [9] as conveying the correspondence between solutions of the 2D PWE, which evolve from Fourier or Hankel-related source functions (according to whether the propagation problem concerns a rectangular or a circular cylindrical geometry), a similar interpretation has been shown to hold for the caloric Appell transformation. It manifests indeed the correspondence between temperature functions arising from "source functions", which are related by a Laplace or a "radial-Laplace"-type transform according to whether the 1D HE or the radial HE is concerned. The optical Appell transformation is a symmetry transformation for the 2D PWE, which can be understood as an evolvinglocation Fourier or Hankel transform operator. Similarly, the caloric Appell transformation is a symmetry transformation for the $\mathrm{HE}$, which can be regarded as an evolving-time Laplace or "radial-Laplace"-type transform operator.

Also, resorting to the fractional versions of both the Fourier/Hankel and Laplace/Hankel-type transforms, one can introduce a fractional Appell transformation in relation to the PWE [9] as well as to the HE, thus displaying a family of symmetry transformations for both equations, parameterized by a continuous parameter.

The analysis has resorted to the Lie-algebra based method, as originally developed in a series of seminal papers by Kalnins, Miller and Boyer [10, 11, 12] in connection with the time-dependent SE. Also, it echoes the analysis mastered in [17], aimed at characterizing transformations between wavefunctions in terms of transformations of the respective source functions.

As earlier noted, the Appell transformation has relevance in other contexts as well. It would be interesting to establish whether it is amenable for an analogous interpretation even when framed in other contexts, like, for instance, that of the Kolmogorov equation, considered in [8].

We conclude saying that other results concerned with the HE can be revisited within the optical context. For instance, the property proven in [6], according to which the Appell transformation is essentially the only transformation mapping solutions of the (in general, $n$-dimensional) HE into solutions, applies to the optical context as well. Thus, every transformation mapping wavefunctions into wavefunctions can be understood as composed of Appell transformations, 
scalings and shifts of all the variables. Leutwiler's result has been restated in [18] by resorting to the ray-matrix formalism of paraxial optics, and accordingly exploiting suitable matrix factorization tools, like, for instance, the possibility of realizing any $2 \times 2$ optical ray-matrix, which ultimately identifies a symmetry operator for the 2D PWE, by an appropriate sequence of freesections and lenses, that then can be rearranged to yield the Appell transformation matrix (2.15).

Since scalings and shifts of the inherent variables do not change the functional form of the functions acted on by them, we can say that the world of the solutions of the 2D PWE can be understood as formed by two classes of functions, which are just mapped one onto the other by the Appell transform. Further solutions can be reached by scalings and/or shifts of both the variables $\xi$ and $\zeta$, without changing evidently the functional form of the original wavefunction.

This is not surprising, since, as noted in [18], the symmetry algebra pertaining to the PWE (2.2) arises from Fourier-similarity related operators, being in fact $\widehat{\mathcal{F}} \widehat{\mathrm{X}} \widehat{\mathcal{F}}^{-1}=-\widehat{\mathrm{P}}$, $\widehat{\mathcal{F}} \widehat{\mathrm{P}} \widehat{\mathcal{F}}^{-1}=\widehat{\mathrm{X}}$, and accordingly $\widehat{\mathcal{F}} \widehat{\mathrm{K}}_{ \pm} \widehat{\mathcal{F}}^{-1}=\widehat{\mathrm{K}}_{\mp}, \widehat{\mathcal{F}} \widehat{\mathrm{K}}_{3} \widehat{\mathcal{F}}^{-1}=-\widehat{\mathrm{K}}_{3}$.

Ultimately, the Appell transformation is a manifestation of such a property.

\section{Acknowledgements}

The author wishes to thank an anonymous referee, who with his/her suggestions, comments and criticisms has greatly improved the paper.

\section{References}

[1] Appell M.P., Sur l'équation $\frac{\partial^{2} z}{\partial x^{2}}-\frac{\partial z}{\partial y}=0$ et la théorie de la chaleur, J. Math. Pure Appl. 8 (1892), $187-216$.

[2] Widder D.V., The heat equation, Pure and Applied Mathematics, Vol. 67, Academic Press, New York London, 1975.

[3] Rosenbloom P.C., Widder D.V., Expansions in terms of heat polynomials and associated functions, Trans. Amer. Math. Soc. 92 (1959), 220-266.

[4] Widder D.V., Analytic solutions of the heat equation, Duke Math. J. 29 (1962), 497-503.

[5] Widder D.V., Expansions in series of homogeneous temperature functions of the first and second kinds, Duke Math. J. 36 (1969), 495-509.

[6] Leutwiler H., On the Appell transformation, in Potential Theory (Prague, 1987), Editors J. Kràl et. al., Plenum Press, New York, 1988, 215-222.

[7] Shimomura K., The determination of caloric morphisms on Euclidean domains, Nagoya Math. J. 158 (2000), $133-166$.

[8] Brzezina M., Appell type transformation for the Kolmogorov operator, Math. Nachr. 169 (1994), 59-67.

[9] Torre A., The Appell transformation for the paraxial wave equation, J. Opt. 13 (2011), 015701, 14 pages.

[10] Kalnins E.G., Miller W. Jr., Lie theory and separation of variables. V. The equation $i U_{t}+U_{x x}=0$ and $i U_{t}+U_{x x}-\left(c / x^{2}\right) U=0$, J. Math. Phys. 15 (1974), 1728-1737.

[11] Boyer C.P., Kalnins E.G., Miller W. Jr., Lie theory and separation of variables. VI. The equation $i U_{t}+\Delta_{2} U=$ 0, J. Math. Phys. 16 (1975), 499-511.

[12] Miller W. Jr., Symmetry and separation of variables, Encyclopedia of Mathematics and its Applications, Vol. 4, Addison-Wesley Publishing Co., Reading, Mass. - London - Amsterdam, 1977.

[13] Wolf K.B., Integral transforms in science and engineering, Mathematical Concepts and Methods in Science and Engineering, Vol. 11, Plenum Press, New York - London, 1979.

[14] Olver P.J., Applications of Lie groups to differential equations, 2nd ed., Graduate Texts in Mathematics, Vol. 107, Springer-Verlag, New York, 1993.

[15] Torre A., A note on the general solution of the paraxial wave equation: a Lie algebra view, J. Opt. A: Pure Appl. Opt. 10 (2008), 055006, 14 pages.

[16] Torre A., Separable-variable solutions of the wave equation from a general type of solutions of the paraxial wave equation, in Proceedings of the International Conference "Days on Diffraction" (May 26-29, 2009, St. Petersburg), 178-183. 
[17] Torre A., Linear and quadratic exponential modulation of the solutions of the paraxial wave equation, J. Opt. 12 (2010), 035701, 11 pages.

[18] Torre A., Appell transformation and symmetry transformations for the paraxial wave equation, J. Opt. 13 (2011), 075710, 12 pages.

[19] Kato T., Perturbation theory for linear operators, 2nd ed., Grundlehren der Mathematischen Wissenschaften, Band 132, Springer-Verlag, Berlin - New York, 1976.

[20] Collins S.A. Jr., Lens-system diffraction integral written in terms of matrix optics, J. Opt. Soc. Amer. A 60 (1970), 1168-1177.

[21] Siegman A.E., Lasers, University Science Books, Mill Valley, CA, 1986.

[22] Ballentine L.E., Quantum mechanics, Prentice Hall, Englewood Cliffs, New Jersey, 1990.

[23] Bandres M.A., Gutiérrez-Vega J.C., Cartesian beams, Opt. Lett. 32 (2007), 3459-3461.

[24] Bandres M.A., Gutiérrez-Vega J.C., Circular beams, Opt. Lett. 33 (2008), 177-179.

[25] Bandres M.A., Gutiérrez-Vega J.C., Elliptical beams, Opt. Expr. 16 (2008), 21087-21092.

[26] Sudarshan E.C.G., Mukunda N., Simon R., Realization of first order optical systems using thin lenses, Opt. Acta 32 (1985), 855-872.

[27] Bandres M.A., Guizar-Sicairos M., Paraxial group, Opt. Lett. 34 (2009), 13-15.

[28] Wei J., Norman E., Lie algebraic solution of linear differential equations, J. Math. Phys. 4 (1963), 575-581. Dattoli G., Gallardo J.C., Torre A., An algebraic view to the operatorial ordering and its applications to optics, Riv. Nuovo Cimento (3) 11 (1988), no. 11, 1-79.

Ban M., Decomposition formulas for $s u(1,1)$ and $s u(2)$ Lie algebras and their applications to quantum optics, J. Opt. Soc. Amer. B 10 (1993), 1347-1359.

[29] Magnus W., Oberhettinger F., Soni R.P., Formulas and theorems for the special functions of mathematical physics, 3rd ed., Die Grundlehren der mathematischen Wissenschaften, Band 52, Springer-Verlag, New York, 1966.

[30] Torre A., A note on the Airy beam in the light of the symmetry algebra based approach, J. Opt. A: Pure Appl. Opt. 11 (2009), 125701, 11 pages.

[31] Berry M.V., Balazs N.L., Nonspreading wave packets, Amer. J. Phys. 47 (1979), 264-267.

[32] Besieris I.M., Shaarawi A.M., Ziolkowski R.W., Nondispersive accelerating wave packets, Amer. J. Phys. 62 (1994), 519-521.

[33] Bandres M.A., Accelerating beams, Opt. Lett. 34 (2009), 3791-3793.

[34] Siviloglou G.A., Christodoulides D.N., Accelerating finite energy Airy beams, Opt. Lett. 32 (2007), $979-981$. Siviloglou G.A., Broky J., Dogariu A., Christodoulides D.N., Observation of accelerating Airy beams, Phys. Rev. Lett. 99 (2007), 2139011, 4 pages.

Siviloglou G.A., Broky J., Dogariu A., Christodoulides D.N., Ballistic dynamics of Airy beams, Opt. Lett. 33 (2008), 207-209.

[35] Besieris I.M., Shaarawi A.M., A note on an accelerating finite energy Airy beam, Opt. Lett. 32 (2007), 2447-2449.

[36] Broky J., Siviloglou G.A., Dogariu A., Christodoulides D.N., Self-healing properties of optical Airy beams, Opt. Expr. 16 (2008), 12880-12891.

[37] Morris J.E., Mazilu M., Baumgartl J., Cizmar T., Dholakia K., Propagation characteristics of Airy beams: dependence upon spatial coherence and wavelength, Opt. Expr. 17 (2009), 13236-13245.

[38] Dai H.T., Sun X.W., Luo D., Liu Y.J., Airy beams generated by binary phase element made of polymerdispersed liquid crystals, Opt. Expr. 17 (2009), 19365-19370.

[39] Baumgartl J., Mazilu M., Dholakia K., Optically mediated particle clearing using Airy wavepackets, Nature Photonics 2 (2008), 675-678.

[40] Ellenbogen T., Voloch-Bloch N., Ganany-Padowicz A., Arie A., Nonlinear generation and manipulation of Airy beams, Nature Photonics 3 (2009), 395-398.

[41] Salandrino A., Christodoulides D.N., Airy plasmon: a nondiffracting surface wave, Opt. Lett. 35 (2010), 2082-2084. 
[42] Mendlovic D., Ozaktas H.M., Fractional Fourier transforms and their optical implementation. I, J. Opt. Soc. Amer. A 10 (1993), 1875-1881.

Mendlovic D., Ozaktas H.M., Fractional Fourier transforms and their optical implementation. II, J. Opt. Soc. Amer. A 10 (1993), 2522-2531.

Ozaktas H.M., Kutay M.A., Mendlovic D., Introduction to the fractional Fourier transform and its applications, in Advances in Imaging and Electron Physics, Vol. 106, Editor P.W. Hawkes, Academic Press, San Diego, 1999, 239-291.

[43] Ozatkas H.M., Zalevsky Z., Kutay M.A., The fractional Fourier transform with applications in optics and signal processing, Wiley, New York, 2001.

[44] Lohmann A.W., Image rotation, Wigner rotation, and the fractional order Fourier transform, J. Opt. Soc. Amer. A 10 (1993), 2181-2186.

Lohmann A.W., Mendlovic D., Zalevsky Z., Fractional transformations in optics, in Progress in Optics, Vol. 38, Editor E. Wolf, Elsevier, Amsterdam, 1997, 263-342.

[45] Torre A., The fractional Fourier transform and some of its applications to optics, in Progress in Optics, Vol. 43, Editor E. Wolf, Elsevier, Amsterdam, 2002, 531-596.

[46] Durnin J., Exact solutions for nondiffracting beams. I. The scalar theory, J. Opt. Soc. Amer. A (1987), 651-654.

Durnin J., Miceli J.J., Eberly J.H., Diffraction-free beams, Phys. Rev. Lett. 58 (1987), 1499-1501.

[47] Sheppard C.J.R., Wilson T., Gaussian beams theory of lenses with annular aperture, IEE J. Microwaves Opt. Acoust. 2 (1978), 105-112,

Gori F., Guattari G., Padovani C., Bessel-Gauss beams, Opt. Comm. 64 (1987), 491-495.

[48] Lohmann A.W., Ein neues Dualitatsprinzip in der Optik, Optik 11 (1954), 478-488.

Lohmann A.W., Duality in optics, Optik 89 (1992), 93-97.

[49] Sheppard C.J.R., Beam duality, with application to generalized Bessel-Gaussian, and Hermite- and Laguerre-Gaussian beams, Opt. Expr. 15 (2009), 3690-3697.

[50] Heaviside O., Electrical papers, The Macmillan Co., New York and London, 1892.

Heaviside O., Electromagnetic theory, The Electrician Printing \& Publishing Co., London, Vol. 1, 1894; Vol. 2, 1899; Vol. 3, 1912.

[51] Nahin P.J., Oliver Heaviside: the life, work, and times of an electrical genius of the victorian age, The Johns Hopkins University Press, Baltimore, 2002.

[52] Tranter C.J., Integral transforms in mathematical physics, Methuen \& Co., Ltd., London; John Wiley \& Sons, Inc., New York, 1951.

[53] Davies B., Integral transforms and their applications, Applied Mathematical Sciences, Vol. 25, SpringerVerlag, New York - Heidelberg, 1978.

[54] Bargmann V., On a Hilbert space of analytic functions and an associated integral transform, Comm. Pure Appl. Math. 14 (1961), 187-214.

Bargmann V., On a Hilbert space of analytic functions and an associated integral transform. II. A family of related function spaces. Application to distribution theory, Comm. Pure Appl. Math. 20 (1967), 1-101.

[55] Moshinsky M., Quesne C., Linear canonical transformations and their unitary representations, J. Math. Phys. 12 (1971), 1772-1780.

[56] Quesne C., Moshinsky M., Canonical transformations and matrix elements, J. Math. Phys. 12 (1971), $1780-1783$.

[57] Wolf K.B., Canonical transforms. I. Complex linear transforms, J. Math. Phys. 15 (1974), 1295-1301.

[58] Wolf K.B., Canonical transforms. II. Complex radial transforms, J. Math. Phys. 15 (1974), $2102-2111$.

[59] Kramer P., Moshinsky M., Seligman T.H., Complex extensions of canonical transformations and quantum mechanics, in Group Theory and Its Applications, Vol. III, Editor E.M. Loebl, Academic Press, New York, 1975, 249-332.

[60] Wolf K.B., Canonical transforms, separation of variables and similarity solutions for a class of parabolic differential equations, J. Math. Phys. 17 (1976), 601-613.

[61] Wolf K.B., On self-reciprocal functions under a class of integral transforms, J. Math. Phys. 18 (1977), 1046-1051.

[62] Namias V., The fractional order Fourier transform and its application to quantum mechanics, J. Inst. Appl. Math. 25 (1980), 241-265. 
[63] Namias V., Fractionalization of Hankel transforms, J. Inst. Math. Appl. 26 (1980), 187-197.

[64] McBride A.C., Kerr F.H., On Namias's fractional Fourier transforms, IMA J. Appl. Math. 39 (1987), 159175.

[65] Pei S.-C., Ding J.-J., Eigenfunctions of linear canonical transform, IEEE Trans. Signal Process. 50 (2002), $11-26$.

[66] Torre A., Linear and radial transforms of fractional order, J. Comp. Appl. Math. 153 (2003), 477-486.

[67] Alieva T., Bastiaans M.J., Properties of the linear canonical integral transformation, J. Opt. Soc. Amer. A 24 (2007), 3658-3665.

[68] Stern A., Uncertainty principles in linear canonical transform domains and some of their implications in optics, J. Opt. Soc. Amer. A 25 (2008), 647-652.

[69] Deng B., Tao R., Wang Y., Convolution theorems for the linear canonical transform and their applications, Sci. China Ser. F 49 (2006), 592-603.

[70] Koç A., Ozaktas H.M., Hesselink L., Fast and accurate algorithm for the computation of complex linear canonical transforms, J. Opt. Soc. Amer. A 27 (2010), 1288-1302.

[71] Sharma K.K., Fractional Laplace transform, Signal Image Video Process 4 (2010), 377-379.

[72] Louck J.D., Moshinsky M., Wolf K.B., Canonical transformations and accidental degeneracy. I. The anisotropic oscillator, J. Math. Phys. 14 (1973), 692-695,

Louck J.D., Moshinsky M., Wolf K.B., Canonical transformations and accidental degeneracy. II. The isotropic oscillator in a sector, J. Math. Phys. 14 (1973), 696-700.

[73] Barut A.O., Girardello L., New "coherent" states associated with non-compact groups, Comm. Math. Phys. 21 (1971), 41-55.

[74] Linares Linares M., Méndez Pérez J.M.R., A Hankel type integral transformation on certain space of distributions, Bull. Calcutta Math. Soc. 83 (1991), 447-546.

Linares Linares M., Méndez Pérez J.M.R., Hankel complementary integral transformations of arbitrary order, Internat. J. Math. Math. Sci. 15 (1992), 323-332.

[75] Malgonde S.P., Debnath L., On Hankel type integral transformations of generalized functions, Integral Transforms Spec. Funct. 15 (2004), 421-430.

[76] Malgonde S.P., Bandewar S.R., Debnath L., Mixed Parseval equation and generalized Hankel-type integral transformation of distributions, Integral Transforms Spec. Funct. 15 (2004), 431-443.

[77] Torre A., Hankel-type integral transforms and their fractionalization: a note, Integral Transforms Spec. Funct. 19 (2008), 277-292.

[78] Bragg L.R., The radial heat polynomials and related functions, Trans. Amer. Math. Soc. 119 (1965), 270290.

Bragg L.R., The radial heat equation and Laplace transforms, SIAM J. Appl. Math. 14 (1966), 986-993. 